\title{
Random Vibration Analysis of Train Moving over Slab Track on Bridge under Track Irregularities and Earthquakes by Pseudoexcitation Method
}

\author{
Zhiping Zeng, ${ }^{1,2,3}$ Kunteng Zhu, ${ }^{1,3}$ Xianfeng He, ${ }^{1}$ Wentao Xu, ${ }^{4}$ \\ Lingkun Chen, 5 and Ping Lou ${ }^{1,3}$ \\ ${ }^{1}$ School of Civil Engineering, Central South University, Changsha 410075, China \\ ${ }^{2}$ Department of Architecture, Kanagawa University, Kanagawa 221-8686, Japan \\ ${ }^{3}$ Key Laboratory of Engineering Structure of Heavy Railway (Central South University), Ministry of Education, \\ Changsha 410075, China \\ ${ }^{4}$ School of Mechanics and Engineering Science, Zhengzhou University, Zhengzhou 450001, China \\ ${ }^{5}$ College of Civil Science and Technology, Yangzhou University, Yangzhou 225127, China
}

Correspondence should be addressed to Kunteng Zhu; 61134963@qq.com

Received 17 March 2015; Revised 22 May 2015; Accepted 25 May 2015

Academic Editor: Luca Guerrini

Copyright (C) 2015 Zhiping Zeng et al. This is an open access article distributed under the Creative Commons Attribution License, which permits unrestricted use, distribution, and reproduction in any medium, provided the original work is properly cited.

This paper investigates the random vibration and the dynamic reliability of operation stability of train moving over slab track on bridge under track irregularities and earthquakes by the pseudoexcitation method (PEM). Each vehicle is modeled by multibody dynamics. The track and bridge is simulated by a rail-slab-girder-pier interaction finite element model. The coupling equations of motion are established based on the wheel-rail interaction relationship. The random excitations of the track irregularities and seismic accelerations are transformed into a series of deterministic pseudoexcitations by PEM. The time-dependent power spectral densities (PSDs) of the random vibration of the system are obtained by step-by-step integration method, and the corresponding dynamic reliability is estimated based on the first-passage failure criterion. A case study is then presented in which a high-speed train moves over a slab track resting on a simply supported girder bridge. The PSD characteristics of the random vibration of the bridge and train are analyzed, the influence of the wheel-rail-bridge interaction models on the random vibration of the bridge and train is discussed, and furthermore the influence of train speed, earthquake intensity, and pier height on the dynamic reliability of train operation stability is studied.

\section{Introduction}

High-speed railway has become one of the most important forms of public transportation in many countries [1-3]. Meanwhile, bridges have been widely used as the supporting structures for high-speed railway $[4,5]$; for example, bridges account for approximately $75 \%$ of the length of the TaipeiKaohsiung high-speed railway in Taiwan [6]. This considerably increases the probability of earthquakes taking place when trains are crossing bridges. As for railway bridges, it is possible that the bridge itself may remain safe during an earthquake but may not be safe enough for the train to move over it due to excessive vibration of the sustaining bridge [7].
Evidently, the dynamic response characteristic and the stability of the moving trains over bridges shaken by earthquakes have become a subject of great concern for the railway engineers [8-11], especially in those countries that are earthquakeprone [12-14]. In the study by Miura [15], the emphasis was placed on the earthquake-induced displacement of track and structures, as well as the damage of trains caused by earthquake excitations, rather than on the dynamic stability of trains during an earthquake. Miyamoto et al. [16] analyzed analytically the operation safety of railway vehicles under the action of earthquakes using a three-dimensional simplified vehicle model, where sine waves were used as the input excitation and the vehicle was assumed to remain stationary on the 
track. Yang et al. [17] investigated the dynamic stability of the train, initially static or traveling over bridges shaken by earthquakes by a three-dimensional model for the train, track, and bridge. In their works, the maximum allowable speeds for the train to run safely under four specific seismic accelerations were evaluated on some threshold values. Matsumoto et al. [18] developed a vehicle/structure dynamic interaction analysis program, known as DIASTARS, to study the operation safety of railway vehicles subjected to earthquake motion. Zhang et al. [19] proposed a method to simulate the dynamic responses of the vehicle-bridge interaction system under multisupport seismic excitation, by which the histories of the dynamic responses were obtained for a high-speed train traversing a steel truss cable-stayed bridge with different seismic intensities and different train speeds. In these pioneering works, most researchers either neglected the track system or took only the conventional ballasted track into consideration. Since the track system is a flexible medium vibrating with the train and bridge, it can affect the extent of interaction between the two subsystems, especially for the train moving in the high-speed range. To the knowledge of the authors, rather few researches have conducted on the random vibrations and the stability of trains running over ballastless track on bridges shaken by earthquake. It follows that the index values computed for the evaluation of the random vibrations and the stability of trains may not be accurate enough for modern high-speed and urban railways, in which the ballastless tracks, for example, slab track, are widely used [20-25]. On the other hand, the track irregularity and seismic motion are usually only treated as one or few time-history samples to compute the dynamic responses of train-track/bridge interaction system in most of the previous literatures. In fact, these results can only be regarded as the particular cases of a sequence of possible outcomes because of the randomness of track irregularity and seismic motion. Therefore it is of great importance to evaluate the random vibrations and the stability of the moving trains on a random vibration basis in order to ensure the reliability of the simulation. Unfortunately, the conventional approach for random vibration analysis [26] is computationally inefficient, especially for the sophisticated train-slab track-bridge interaction (TSTBI) system. Therefore more efficient and accurate algorithm should be employed to analyze the random vibrations of the TSTBI system as well as the stability of the moving trains, such as PEM [6, 27-29]. Zhang et al. [6] have investigated the random vibrations for the train-bridge system subjected to horizontal earthquake by PEM. However, the track was assumed to be attached firmly to the girder, and the wheel displacements were assumed to be fully constrained by the girder displacements and track irregularities; that is, the inherent relative displacements between the wheel, the track, and the girder were not taken into consideration.

In present paper, a three-dimensional TSTBI model is constructed based on the assumptions made for modeling such a system. The equations of motion for the major components of the model, that is, the vehicle, rail, slab, girder, and pier, are formulated by means of the finite element method (FEM) and energy principle [30], and, using these, the equations of motion for the entire TSTBI system are assembled.
The track irregularities are regarded as a series of uniformly modulated, multipoint, different-phase random excitations by taking the time lags between the wheels into account, while the earthquakes are assumed as a series of uniformly modulated, nonstationary, evolutionary random excitations. Thus, the random excitations caused by the track irregularities and earthquakes are transformed into a series of deterministic pseudoharmonic excitation vectors according to PEM and the wheel-rail interaction relationship, so that the timedependent PSDs of the random vibration responses of the system excited by track irregularities and earthquakes can be obtained by step-by-step integration method such as Wilson$\theta$ method. A high-speed train consisting of eight vehicles moving over a slab track resting on a fifteen-span simply supported girder bridge is presented. Firstly, the PSD characteristics of the random dynamic responses of the bridge and train are investigated. Secondly, the influence of the wheel-railbridge interaction models on the random vibration characteristic of the bridge and train are studied. Thirdly, the influence of the train speed, the earthquake intensity, and the pier height on the dynamic reliability of train operation stability is analyzed. Finally, some useful conclusions are drawn.

\section{Three-Dimensional Models of Train, Slab Track, and Bridge}

Figure 1 depicts a train consisting of a series of four-axle vehicles moving with a constant speed $v$ on a slab track resting on a simply supported girder bridge shaken by the track irregularities and earthquakes. For the sake of simplicity, it is assumed that no inelastic deformation occurs on each subsystem during earthquakes.

2.1. Model of Train. The train consists of the rear and front motor cars numbered 1 and 2, respectively, and $N_{v}$ trailer cars numbered $1,2, \ldots, N_{v}$ from left to right.

Each trailer car in the train is modeled as a mass-springdamper system consisting of one carbody, two bogies, four wheelsets, and a two-stage suspension system. As shown in Figure 1, the carbody rests on the front and rear bogies, each of which in turn is supported by two wheelsets. The carbody is modeled as a rigid body with mass $m_{c}$ and three moments of inertia $I_{c x}, I_{c y}$, and $I_{c z}$ about the $x$-axis, $y$-axis, and $z$ axis through its center of gravity. Similarly, each bogie is considered as a rigid body with mass $m_{t}$ and three moments of inertia $I_{t x}, I_{t y}$, and $I_{t z}$. Each wheelset is considered as a rigid body with mass $m_{w}$ and two moments of inertia $I_{w x}$ and $I_{w z}$. The secondary suspension between the carbody and each bogie is characterized by a three-dimensional springdamper system with stiffness $k_{s x}, k_{s y}$, and $k_{s z}$ and damping coefficients $c_{s x}, c_{s y}$, and $c_{s z}$, respectively. Likewise the springs and shock absorbers in the primary suspension for each wheelset are characterized by $k_{p x}, k_{p y}$, and $k_{p z}$ and $c_{p x}$, $c_{p y}$, and $c_{p z}$, respectively. By neglecting of the longitudinal motions, the motions of the $j$ th trailer carbody with respect to its center of gravity may be described by the lateral displacement $y_{c j}$, the vertical displacement $z_{c j}$, the rolling displacement $\theta_{c j}$, the pitching displacement $\varphi_{c j}$, and the yawing displacement $\psi_{c j}$, where the subscript $j$ denotes the trailer 


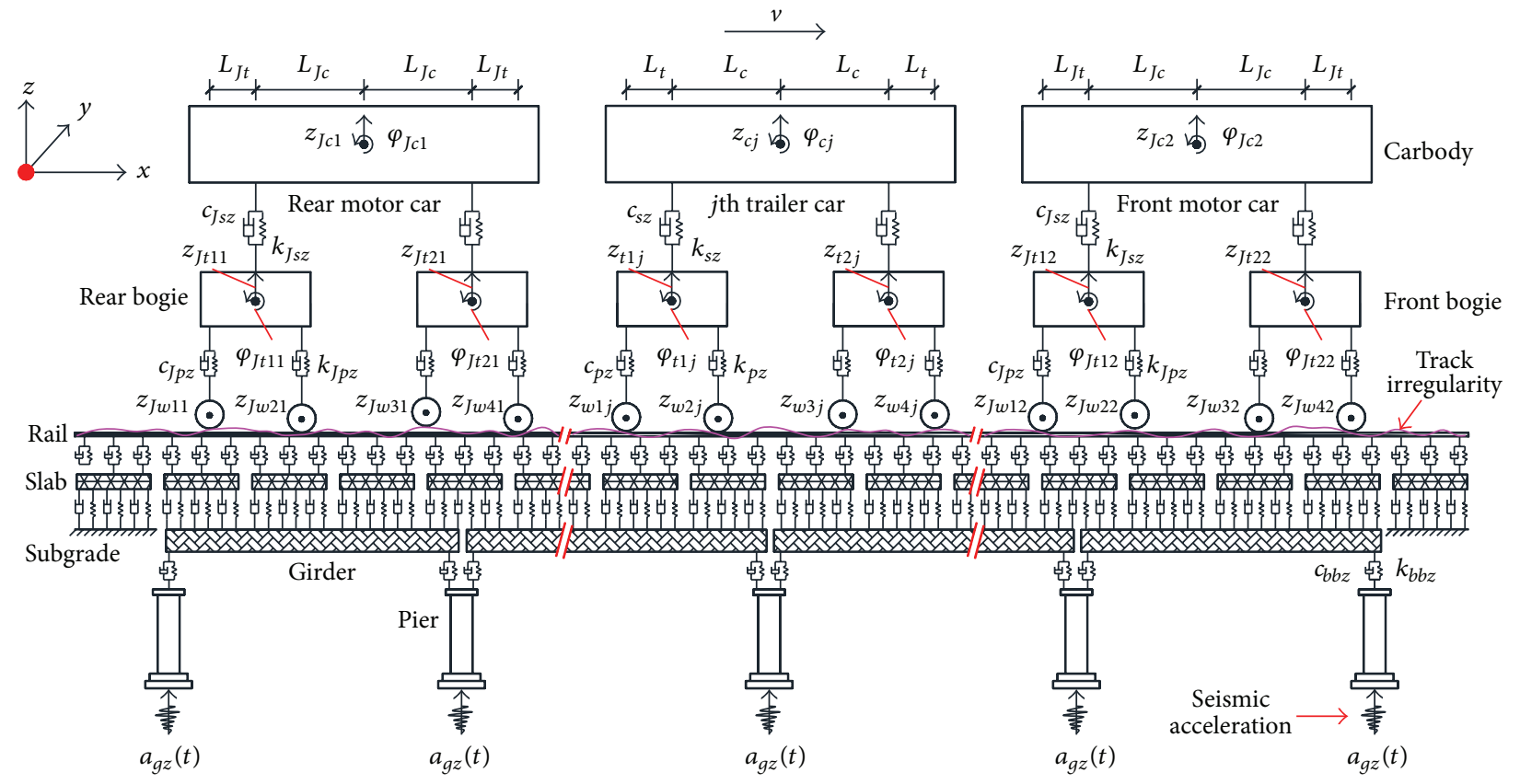

(a)

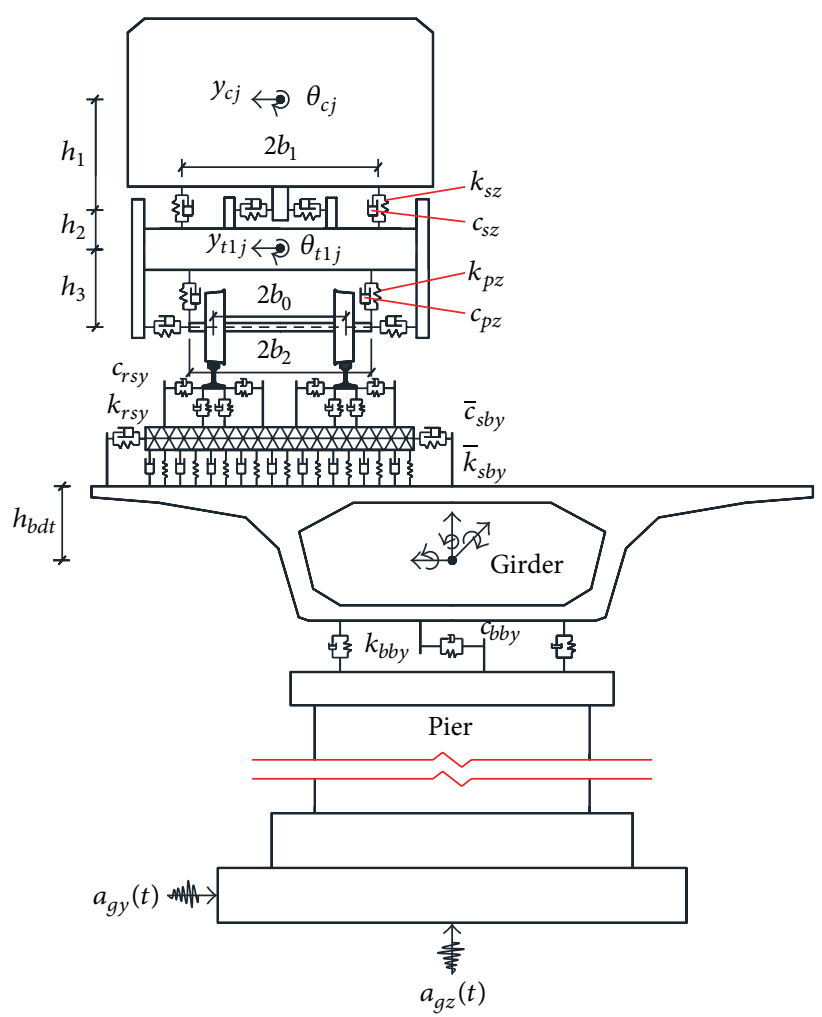

(b)

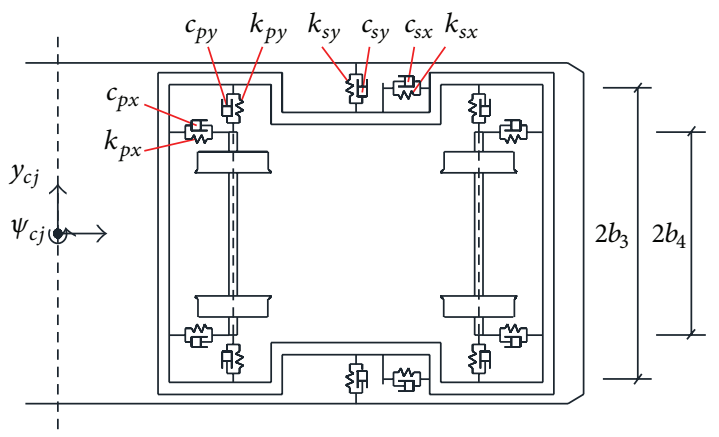

(c)

FIgURE 1: Three-dimensional model for the TSTBI system. (a) Frontal view. (b) Left side view (the jth trailer car, track, and bridge). (c) Top view (half of the $j$ th trailer car without track and bridge).

car number. Similarly, the motions of both the rear and front bogies of the $j$ th trailer car may be also described, respectively, by $y_{t 1 j}, z_{t 1 j}, \theta_{t 1 j}, \varphi_{t 1 j}$, and $\psi_{t 1 j}$ and $y_{t 2 j}, z_{t 2 j}$, $\theta_{t 2 j}, \varphi_{t 2 j}$, and $\psi_{t 2 j}$. The motion from left to right of the $h$ th
( $h=1-4)$ wheelset of the $j$ th trailer car may be described by $y_{\mathrm{wh} j}, z_{\mathrm{wh} j}, \theta_{\mathrm{wh} j}$, and $\psi_{\mathrm{wh} j}$, respectively. Therefore, the total number of DOFs for each trailer car is 31 . However, it is assumed that no jump occurs between each wheel of all 
vehicles and the rail in this paper; that is, the vertical and rolling displacements of each wheelset are constrained by the displacements of the rail. Consequently, the independent DOFs for each trailer car become 23.

Similarly, each motor carbody has mass $m_{J c}$ and three moments of inertia $I_{J c x}, I_{J c y}$, and $I_{J c z}$. Each bogie has mass $m_{J t}$ and three moments of inertia $I_{t x}, I_{t y}$, and $I_{t z}$. Each wheelset has mass $m_{J w}$ and two moments of inertia $I_{J w x}$ and $I_{J w z}$. The secondary suspension is characterized by springs with stiffness $k_{J x x}, k_{J s y}$, and $k_{J s z}$ and dampers with damping coefficients $c_{J s x}, \mathcal{c}_{J s y}$, and $c_{J s z}$. The primary suspension is characterized by springs with stiffness $k_{J p x}, k_{J p y}$, and $k_{J p z}$ and dampers with damping coefficients $c_{J p x}$, $c_{J p y}$, and $c_{J p z}$. The DOFs of the carbody are denoted as $y_{J c i}, z_{J c i}, \theta_{J c i}, \varphi_{J c i}$, and $\psi_{J c i}$. The DOFs of the rear bogie are denoted as $y_{J t 1 i}, z_{J t 1 i}$, $\theta_{I t 1 i}, \varphi_{I t 1 i}$, and $\psi_{I t 1 i}$. The DOFs of the front bogie are denoted as $y_{I t 2 i}, z_{I t 2 i}, \theta_{I t 2 i}, \varphi_{I t 2 i}$, and $\psi_{I t 2 i}$. The DOFs of the four wheelsets are denoted as $y_{J w 1 i}-y_{J w 4 i}$ and $\psi_{J w 1 i}-\psi_{J w 4 i}$. Herein the subscript $i$ denotes the motor car number.

2.2. Models of Slab Track and Bridge. As shown in Figure 1, the rail, slab, girder, and pier are all modeled as elastic Bernoulli-Euler beams. On the basis of FEM, the rail, slab, girder, and pier are all divided into a series of beam elements. The lateral elasticity and damping properties of the fastener are represented by discrete massless springs with stiffness $k_{r s y}$ and dampers with damping coefficient $c_{r s y}$. The vertical elasticity and damping properties of the fastener are represented by $k_{r s z}$ and $c_{r s z}$. The lateral elasticity and damping properties of the cement asphalt mortar (CAM) beneath the slab are represented by continuous massless springs with stiffness $\bar{k}_{s b y}$ and dampers with damping coefficient $\bar{c}_{s b y}$. The vertical elasticity and damping properties of CAM are represented by $\bar{k}_{s b z}$ and $\bar{c}_{s b z}$. The elasticity and damping properties of the bridge bearing are represented by massless springs with stiffness $k_{b b y}, k_{b b z}$, and $k_{b b \theta}$ and dampers with damping coefficients $c_{b b y}, c_{b b z}$, and $c_{b b \theta}$. In addition, the damping property of the beams is assumed to be of the Rayleigh type [17]. By neglecting the longitudinal motion, each node of the rail, slab, and girder has five DOFs, that is, lateral displacement, vertical displacement, and rotations about the $x$-axis, $y$-axis, and $z$ axis. Each node of the pier has three DOFs, that is, lateral displacement, vertical displacement, and rotation about the $x$-axis.

\section{Equations of Motion for the TSTBI System}

By using the energy principle [30], one can derive the threedimensional equations of motion written in submatrix form for the TSTBI system as

$$
\begin{gathered}
{\left[\begin{array}{ccccc}
\mathbf{M}_{t t} & 0 & 0 & 0 & 0 \\
0 & \mathbf{M}_{r r} & 0 & 0 & 0 \\
0 & 0 & \mathbf{M}_{s s} & 0 & 0 \\
0 & 0 & 0 & \mathbf{M}_{b b} & 0 \\
0 & 0 & 0 & 0 & \mathbf{M}_{p p}
\end{array}\right]\left[\begin{array}{l}
\ddot{\mathbf{X}}_{t} \\
\ddot{\mathbf{X}}_{r} \\
\ddot{\mathbf{X}}_{s} \\
\ddot{\mathbf{X}}_{b} \\
\ddot{\mathbf{X}}_{p}
\end{array}\right]} \\
+\left[\begin{array}{ccccc}
\mathbf{C}_{t t} & \mathbf{C}_{t r} & 0 & 0 & 0 \\
\mathbf{C}_{r t} & \mathbf{C}_{r r} & \mathbf{C}_{r s} & 0 & 0 \\
0 & \mathbf{C}_{s r} & \mathbf{C}_{s s} & \mathbf{C}_{s b} & 0 \\
0 & 0 & \mathbf{C}_{b s} & \mathbf{C}_{b b} & \mathbf{C}_{b p} \\
0 & 0 & 0 & \mathbf{C}_{p b} & \mathbf{C}_{p p}
\end{array}\right]\left[\begin{array}{l}
\dot{\mathbf{X}}_{t} \\
\dot{\mathbf{X}}_{r} \\
\dot{\mathbf{X}}_{s} \\
\dot{\mathbf{X}}_{b} \\
\dot{\mathbf{X}}_{p}
\end{array}\right] \\
+\left[\begin{array}{ccccc}
\mathbf{K}_{t t} & \mathbf{K}_{t r} & 0 & 0 & 0 \\
\mathbf{K}_{r t} & \mathbf{K}_{r r} & \mathbf{K}_{r s} & 0 & 0 \\
0 & \mathbf{K}_{s r} & \mathbf{K}_{s s} & \mathbf{K}_{s b} & 0 \\
0 & 0 & \mathbf{K}_{b s} & \mathbf{K}_{b b} & \mathbf{K}_{b p} \\
0 & 0 & 0 & \mathbf{K}_{p b} & \mathbf{K}_{p p}
\end{array}\right]\left[\begin{array}{l}
\mathbf{X}_{t} \\
\mathbf{X}_{r} \\
\mathbf{X}_{s} \\
\mathbf{X}_{b} \\
\mathbf{X}_{p}
\end{array}\right]=\left[\begin{array}{l}
\mathbf{F}_{t} \\
\mathbf{F}_{r} \\
\mathbf{F}_{s} \\
\mathbf{F}_{b} \\
\mathbf{F}_{p}
\end{array}\right],
\end{gathered}
$$

where the subscripts " $t$, , " $r$, , " $s$," " $b$," and " $p$ " denote the train, rail, slab, girder, and pier, respectively. The displacement vector, mass matrix, stiffness matrix, damping matrix, and the load vector of the train, rail, slab, girder, and pier are explained as follows briefly, and the detailed derivation can refer to $[30,31]$.

3.1. Displacement Vectors for Tran, Rail, Slab, Girder, and Pier. The total train displacement vector $\mathbf{X}_{t}$ of order $T_{\text {dof }} \times 1\left(T_{\text {dof }}=\right.$ $23 \times N_{v}+23 \times 2$ ) can be written as

$$
\mathbf{X}_{t}=\left[\begin{array}{llllll}
\mathbf{X}_{J 1} & \mathbf{X}_{v 1} & \mathbf{X}_{v 2} & \cdots & \mathbf{X}_{v N_{v}} & \mathbf{X}_{J 2}
\end{array}\right]^{\mathbf{T}},
$$

where the superscript " $T$ " denotes the transpose of the matrix and $\mathbf{X}_{v j}\left(j=1,2, \ldots, N_{v}\right)$ and $\mathbf{X}_{J i}(i=1,2)$ the displacement vectors of the $j$ th trailer car and the $i$ th motor car, respectively. These can be expressed as

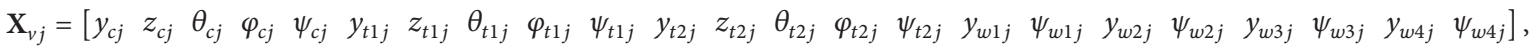

$$
\begin{aligned}
& \mathbf{X}_{I i} \\
& =\left[\begin{array}{lllllllllllllllllllllll}
y_{J c i} & z_{J c i} & \theta_{J c i} & \varphi_{I c i} & \psi_{J c i} & y_{J t 1 i} & z_{J t 1 i} & \theta_{J t 1 i} & \varphi_{J t 1 i} & \psi_{J t 1 i} & y_{J t 2 i} & z_{J t 2 i} & \theta_{J t 2 i} & \varphi_{J t 2 i} & \psi_{J t 2 i} & y_{J w 1 i} & \psi_{J w 1 i} & y_{J w 2 i} & \psi_{J w 2 i} & y_{J w 3 i} & \psi_{J w 3 i} & y_{J w 4 i} & \psi_{J w 4 i}
\end{array}\right] .
\end{aligned}
$$

The displacement vector of the rail $\mathbf{X}_{r}$ of order $2 \bar{N}_{r} \times 1$, comprising the displacement vectors $\mathbf{X}_{L r}$ of the left rail and $\mathbf{X}_{R r}$ of the right rail, both of order $\bar{N}_{r} \times 1$, can be written as

$$
\begin{aligned}
\mathbf{X}_{r} & =\left[\begin{array}{ll}
\mathbf{X}_{L r} & \mathbf{X}_{R r}
\end{array}\right]^{\mathrm{T}}, \\
\mathbf{X}_{L r} & =\mathbf{X}_{R r}=\left[\begin{array}{llll}
q_{r 1} & q_{r 2} & \cdots & q_{r \bar{N}_{r}}
\end{array}\right],
\end{aligned}
$$


where $\bar{N}_{r}$ denotes the total number of DOFs of each rail.

Similarly, the displacement vector of the slab $\mathbf{X}_{s}$ of order $\bar{N}_{s} \times 1$, the displacement vector of the girder $\mathbf{X}_{b}$ of order $\bar{N}_{b} \times 1$, and the displacement vector of the pier $\mathbf{X}_{p}$ of order $\bar{N}_{p} \times 1$ can be written, respectively, as

$$
\begin{aligned}
& \mathbf{X}_{s}=\left[\begin{array}{llll}
\mathbf{X}_{s 1} & \mathbf{X}_{s 2} & \cdots & \mathbf{X}_{s N_{s}}
\end{array}\right]^{\mathbf{T}}, \\
& \mathbf{X}_{b}=\left[\begin{array}{llll}
\mathbf{X}_{b 1} & \mathbf{X}_{b 2} & \cdots & \mathbf{X}_{b N_{b}}
\end{array}\right]^{\mathbf{T}}, \\
& \mathbf{X}_{p}=\left[\begin{array}{llll}
\mathbf{X}_{p 1} & \mathbf{X}_{p 2} & \cdots & \mathbf{X}_{p N_{p}}
\end{array}\right]^{\mathbf{T}},
\end{aligned}
$$

where $\mathbf{X}_{s i}\left(i=1,2, \ldots, N_{s}\right)$ denotes the displacement vector of the $i$ th slab, $N_{s}$ the total number of slabs, and $\bar{N}_{s}$ the total number of DOFs of all slabs; $\mathbf{X}_{b i}\left(i=1,2, \ldots, N_{b}\right)$ denotes the displacement vector of the $i$ th girder, $N_{b}$ the total number of girders, and $\bar{N}_{b}$ the total number of DOFs of all girders; and $\mathbf{X}_{p i}\left(i=1,2, \ldots, N_{p}\right)$ denotes the displacement vector of the $i$ th pier, $N_{p}$ the total number of piers, and $\bar{N}_{p}$ the total number of DOFs of all piers.

3.2. Matrices for Train, Rail, Slab, Girder, and Pier. The matrices of the train are marked with the subscript " $t t$." The mass matrix $\mathbf{M}_{t t}$, the stiffness matrix $\mathbf{K}_{t t}$, and the damping matrix $\mathbf{C}_{t t}$ of the train, all of order $\left(23 \times N_{v}+23 \times 2\right) \times$ $\left(23 \times N_{v}+23 \times 2\right)$, can be written, respectively, as

$$
\begin{aligned}
\mathbf{M}_{t t} & =\operatorname{diag}\left[\begin{array}{llllll}
\mathbf{M}_{J 1} & \mathbf{M}_{v 1} & \mathbf{M}_{v 2} & \cdots & \mathbf{M}_{v N_{v}} & \mathbf{M}_{J 2}
\end{array}\right], \\
\mathbf{K}_{t t} & =\operatorname{diag}\left[\begin{array}{lllllll}
\mathbf{K}_{J 1} & \mathbf{K}_{v 1} & \mathbf{K}_{v 2} & \cdots & \mathbf{K}_{v N_{v}} & \mathbf{K}_{J 2}
\end{array}\right], \\
\mathbf{C}_{t t} & =\operatorname{diag}\left[\begin{array}{lllllll}
\mathbf{C}_{J 1} & \mathbf{C}_{v 1} & \mathbf{C}_{v 2} & \cdots & \mathbf{C}_{v N_{v}} & \mathbf{C}_{J 2}
\end{array}\right],
\end{aligned}
$$

where $\mathbf{M}_{v j}, \mathbf{K}_{v j}$, and $\mathbf{C}_{v j}$, all of order $23 \times 23$, denote the mass, stiffness, and damping matrices of the $j$ th trailer car, respectively; $\mathbf{M}_{J i}, \mathbf{K}_{J i}$, and $\mathbf{C}_{J i}$, all of order $23 \times 23$, denote the mass, stiffness, and damping matrices of the $i$ th motor car, respectively.

The matrices of the rail are marked with the subscript " $r r$." The mass matrix $\mathbf{M}_{r r}$ of the rail with order $2 \bar{N}_{r} \times 2 \bar{N}_{r}$, comprising mass matrices $\mathbf{M}_{L r r}$ of the left rail and $\mathbf{M}_{R r r}$ of the right rail, both of order $\bar{N}_{r} \times \bar{N}_{r}$, can be written as

$$
\mathbf{M}_{r r}=\operatorname{diag}\left[\begin{array}{ll}
\mathbf{M}_{L r r} & \mathbf{M}_{R r r}
\end{array}\right] \text {. }
$$

The stiffness matrix $\mathbf{K}_{r r}$ of the rail of order $2 \bar{N}_{r} \times 2 \bar{N}_{r}$, which is composed of the stiffness matrices of the left and right rails $\mathbf{K}_{L r r}$ and $\mathbf{K}_{R r r}$, both of order $\bar{N}_{r} \times \bar{N}_{r}$, and the left rail-right rail interaction stiffness matrices $\mathbf{K}_{L r R r}$ and $\mathbf{K}_{R r L r}$ of $\operatorname{order} \bar{N}_{r} \times \bar{N}_{r}$, can be written as

$$
\mathbf{K}_{r r}=\left[\begin{array}{cc}
\mathbf{K}_{L r r} & \mathbf{K}_{L r R r} \\
\mathbf{K}_{R r L r} & \mathbf{K}_{R r r}
\end{array}\right],
$$

where the stiffness matrices $\mathbf{K}_{L r R r}$ and $\mathbf{K}_{R r L r}$ are induced by the train's weight acting upon the rails by the wheelsets.

The damping matrix $\mathbf{C}_{r r}$ of the rail of order $2 \bar{N}_{r} \times 2 \bar{N}_{r}$, consisting of the damping matrices of the left and right rails
$\mathrm{C}_{L r r}$ and $\mathrm{C}_{R r r}$, respectively, both of order $\bar{N}_{r} \times \bar{N}_{r}$, and can be written as

$$
\mathbf{C}_{r r}=\operatorname{diag}\left[\begin{array}{ll}
\mathbf{C}_{L r r} & \mathbf{C}_{R r r}
\end{array}\right] .
$$

The matrices of the slab, girder, and pier, marked with the subscripts "ss," " $b b$," and " $p p$," respectively, are not given here but can be derived by following a procedure similar to that given above.

3.3. Matrices for Train-Rail-Slab-Girder-Pier Interaction. The matrices for the train-rail interaction, marked with subscripts " $t r$ " or " $r t$," consist of the train-left rail interaction matrices marked with subscript " $t L r$," and the train-right rail interaction matrices marked with subscript " $t R r$." The stiffness matrices $\mathbf{K}_{t r}$ and $\mathbf{K}_{r t}$ of order $T_{\text {dof }} \times 2 \bar{N}_{r}$ and damping matrices $\mathbf{C}_{t r}$ and $\mathbf{C}_{r t}$ of order $T_{\text {dof }} \times 2 \bar{N}_{r}$, describing the trainrail interaction, can be written according to the vertical and lateral wheel-rail interaction relationships $[32,33]$ as

$$
\begin{aligned}
& \mathbf{K}_{t r}=\left[\begin{array}{ll}
\mathbf{K}_{t L r} & \mathbf{K}_{t R r}
\end{array}\right]_{T_{\mathrm{dof}} \times 2 N_{r}}, \\
& \mathbf{C}_{t r}=\left[\begin{array}{ll}
\mathbf{C}_{t L r} & \mathbf{C}_{t R r}
\end{array}\right]_{T_{\text {dof } \times 2 N_{r}}}, \\
& \mathbf{K}_{r t}=\mathbf{K}_{t r}^{\mathbf{T}}, \\
& \mathbf{C}_{r t}=\mathbf{C}_{t r}^{\mathbf{T}} .
\end{aligned}
$$

The matrices for the rail-slab interaction, marked with subscripts " $r s$ " or "sr", are induced by the stiffness and damping of the fastener between the rail and slab. The matrices for the slab-girder interaction, marked with subscripts " $s$ " or " $b s$," are induced by the stiffness and damping of the CAM between the slab and girder. The matrices for the girder-pier interaction, marked with subscripts " $b p$ " or " $p b$," are induced by the stiffness and damping of the bearing between the girder and pier. All of them can be derived similarly.

3.4. Load Vectors for Train, Rail, Slab, Girder, and Pier. The load vector $\mathbf{F}_{t}$ of the train of order $T_{\text {dof }} \times 1$ can be written as

$$
\mathbf{F}_{t}=\mathbf{F}_{t}^{1 V}+\mathbf{F}_{t}^{2 V}+\mathbf{F}_{t}^{1 C}+\mathbf{F}_{t}^{2 C}+\mathbf{F}_{t}^{1 A}+\mathbf{F}_{t}^{2 A}+\mathbf{F}_{t}^{2 G}
$$

with

$$
\begin{aligned}
& \mathbf{F}_{t}^{1 V}=\left[\begin{array}{llllll}
\mathbf{F}_{J 1}^{1 V} & \mathbf{F}_{v 1}^{1 V} & \mathbf{F}_{v 2}^{1 V} & \cdots & \mathbf{F}_{v N_{v}}^{1 V} & \mathbf{F}_{J 2}^{1 V}
\end{array}\right]^{\mathbf{T}}, \\
& \mathbf{F}_{t}^{1 C}=\left[\begin{array}{llllll}
\mathbf{F}_{J 1}^{1 C} & \mathbf{F}_{v 1}^{1 C} & \mathbf{F}_{v 2}^{1 C} & \cdots & \mathbf{F}_{v N_{v}}^{1 C} & \mathbf{F}_{J 2}^{1 C}
\end{array}\right]^{\mathbf{T}}, \\
& \mathbf{F}_{t}^{1 A}=\left[\begin{array}{llllll}
\mathbf{F}_{J 1}^{1 A} & \mathbf{F}_{v 1}^{1 A} & \mathbf{F}_{v 2}^{1 A} & \cdots & \mathbf{F}_{v N_{v}}^{1 A} & \mathbf{F}_{J 2}^{1 A}
\end{array}\right]^{\mathbf{T}}, \\
& \mathbf{F}_{t}^{2 V}=\left[\begin{array}{llllll}
\mathbf{F}_{J 1}^{2 V} & \mathbf{F}_{v 1}^{2 V} & \mathbf{F}_{v 2}^{2 V} & \cdots & \mathbf{F}_{v N_{v}}^{2 V} & \mathbf{F}_{J 2}^{2 V}
\end{array}\right]^{\mathbf{T}}, \\
& \mathbf{F}_{t}^{2 C}=\left[\begin{array}{llllll}
\mathbf{F}_{J 1}^{2 C} & \mathbf{F}_{v 1}^{2 C} & \mathbf{F}_{v 2}^{2 C} & \cdots & \mathbf{F}_{v N_{v}}^{2 C} & \mathbf{F}_{J 2}^{2 C}
\end{array}\right]^{\mathbf{T}}, \\
& \mathbf{F}_{t}^{2 A}=\left[\begin{array}{llllll}
\mathbf{F}_{J 1}^{2 A} & \mathbf{F}_{v 1}^{2 A} & \mathbf{F}_{v 2}^{2 A} & \cdots & \mathbf{F}_{v N_{v}}^{2 A} & \mathbf{F}_{J 2}^{2 A}
\end{array}\right]^{\mathbf{T}}, \\
& \mathbf{F}_{t}^{2 G}=\left[\begin{array}{llllll}
\mathbf{F}_{J 1}^{2 G} & \mathbf{F}_{v 1}^{2 G} & \mathbf{F}_{v 2}^{2 G} & \cdots & \mathbf{F}_{v N_{v}}^{2 G} & \mathbf{F}_{J 2}^{2 G}
\end{array}\right]^{\mathbf{T}},
\end{aligned}
$$


where $\mathbf{F}_{t}^{1 V}, \mathbf{F}_{t}^{1 C}$, and $\mathbf{F}_{t}^{1 A}$ represent the load vectors caused by the track elevation, cross level, and alignment irregularities, respectively; $\mathbf{F}_{t}^{2 V}, \mathbf{F}_{t}^{2 C}, \mathbf{F}_{t}^{2 A}$, and $\mathbf{F}_{t}^{2 G}$ represent the load vectors caused by the velocity of the track elevation, cross level, alignment, and gauge irregularities, respectively.

$\mathbf{F}_{v j}^{1 V}, \mathbf{F}_{v j}^{1 C}, \mathbf{F}_{v j}^{1 A}, \mathbf{F}_{v j}^{2 V}, \mathbf{F}_{v j}^{2 C}, \mathbf{F}_{v j}^{2 A}$, and $\mathbf{F}_{v j}^{2 G}$ for the $j$ th trailer car can be written, respectively, as

$$
\begin{aligned}
& \mathbf{F}_{v j}^{1 V}=\sum_{h=1}^{4} k_{p z} r\left(x_{j h}^{V}\right) R_{v h}^{V}, \\
& \mathbf{F}_{v j}^{1 C}=\sum_{h=1}^{4} k_{p z} r\left(x_{j h}^{C}\right) R_{v h}^{C}, \\
& \mathbf{F}_{v j}^{1 A}=\sum_{h=1}^{4} \frac{W_{\mathrm{axle}} \lambda}{b_{0}} r\left(x_{j h}^{A}\right) R_{v h}^{A}, \\
& \mathbf{F}_{v j}^{2 V}=\sum_{h=1}^{4} c_{p z} \dot{r}\left(x_{j h}^{V}\right) R_{v h}^{V}, \\
& \mathbf{F}_{v j}^{2 C}=\sum_{h=1}^{4} c_{p z} \dot{r}\left(x_{j h}^{C}\right) R_{v h}^{C}, \\
& \mathbf{F}_{v j}^{2 A}=\sum_{h=1}^{4}\left(f_{L j h}^{22}+f_{R j h}^{22}\right) \dot{r}\left(x_{j h}^{A}\right) R_{v h}^{A}, \\
& \mathbf{F}_{v j}^{2 G}=\sum_{h=1}^{4} \frac{1}{2}\left(f_{L j h}^{22}-f_{R j h}^{22}\right) \dot{r}\left(x_{j h}^{G}\right) R_{v h}^{A}, \\
& R_{v 1}^{V}=\left[\begin{array}{lllllllllllllllllllllll}
0 & 0 & 0 & 0 & 0 & 0 & 2 & 0 & 2 L_{t} & 0 & 0 & 0 & 0 & 0 & 0 & 0 & 0 & 0 & 0 & 0 & 0 & 0 & 0
\end{array}\right], \\
& R_{v 2}^{V}=\left[\begin{array}{lllllllllllllllllllllll}
0 & 0 & 0 & 0 & 0 & 0 & 2 & 0 & -2 L_{t} & 0 & 0 & 0 & 0 & 0 & 0 & 0 & 0 & 0 & 0 & 0 & 0 & 0 & 0
\end{array}\right], \\
& R_{v 3}^{V}=\left[\begin{array}{lllllllllllllllllllllll}
0 & 0 & 0 & 0 & 0 & 0 & 0 & 0 & 0 & 0 & 0 & 2 & 0 & 2 L_{t} & 0 & 0 & 0 & 0 & 0 & 0 & 0 & 0 & 0
\end{array}\right], \\
& R_{v 4}^{V}=\left[\begin{array}{lllllllllllllllllllllll}
0 & 0 & 0 & 0 & 0 & 0 & 0 & 0 & 0 & 0 & 0 & 2 & 0 & -2 L_{t} & 0 & 0 & 0 & 0 & 0 & 0 & 0 & 0 & 0
\end{array}\right], \\
& R_{v 1}^{C}=R_{v 2}^{C}=\left[\begin{array}{lllllllllllllllllllllll}
0 & 0 & 0 & 0 & 0 & 0 & 0 & b_{2} & 0 & 0 & 0 & 0 & 0 & 0 & 0 & 0 & 0 & 0 & 0 & 0 & 0 & 0 & 0
\end{array}\right], \\
& R_{v 3}^{C}=R_{v 4}^{C}=\left[\begin{array}{lllllllllllllllllllllll}
0 & 0 & 0 & 0 & 0 & 0 & 0 & 0 & 0 & 0 & 0 & 0 & b_{2} & 0 & 0 & 0 & 0 & 0 & 0 & 0 & 0 & 0 & 0
\end{array}\right], \\
& R_{v 1}^{A}=\left[\begin{array}{lllllllllllllllllllllll}
0 & 0 & 0 & 0 & 0 & 0 & 0 & 0 & 0 & 0 & 0 & 0 & 0 & 0 & 0 & 1 & 0 & 0 & 0 & 0 & 0 & 0 & 0
\end{array}\right], \\
& R_{v 2}^{A}=\left[\begin{array}{lllllllllllllllllllllll}
0 & 0 & 0 & 0 & 0 & 0 & 0 & 0 & 0 & 0 & 0 & 0 & 0 & 0 & 0 & 0 & 0 & 1 & 0 & 0 & 0 & 0 & 0
\end{array}\right], \\
& R_{v 3}^{A}=\left[\begin{array}{lllllllllllllllllllllll}
0 & 0 & 0 & 0 & 0 & 0 & 0 & 0 & 0 & 0 & 0 & 0 & 0 & 0 & 0 & 0 & 0 & 0 & 0 & 1 & 0 & 0 & 0
\end{array}\right], \\
& R_{v 4}^{A}=\left[\begin{array}{lllllllllllllllllllllll}
0 & 0 & 0 & 0 & 0 & 0 & 0 & 0 & 0 & 0 & 0 & 0 & 0 & 0 & 0 & 0 & 0 & 0 & 0 & 0 & 0 & 1 & 0
\end{array}\right],
\end{aligned}
$$

where $r\left(x_{j h}^{V}\right), r\left(x_{j h}^{C}\right), r\left(x_{j h}^{A}\right)$, and $r\left(x_{j h}^{G}\right)$ denote the track elevation, cross level, alignment, and gauge irregularities, respectively, at the $h$ th wheel-rail contact point of the $j$ th trailer car; $\dot{r}(\cdot)$ is the first derivative of track irregularity $r(\cdot) ; b_{0}$ is half of the transverse distance between the contact points of the wheel and rail; $b_{2}$ is half of the transverse distance between the vertical primary suspension systems (Figure 1(b)); $L_{t}$ is half of the bogie axle base; $W_{\text {axle }}$ is the axle weight; $\lambda$ is the slope of the wheel tread; and $f_{L j h}^{22}$ and $f_{R j h}^{22}(h=1-4)$ are the lateral creepage coefficients between the $h$ th wheelset of the $j$ th trailer car and the left and right rails, respectively. The lateral creepage coefficient is a function of the wheel-rail normal contact force and the wheel-rail curvature radius at the contact point. In order to make the calculation easier, the coefficient is considered as a constant for certain vehicle by taking the wheel-rail normal contact force as the static wheel 
weight and assuming that the wheel-rail contact point lies in a cone surface for the wheel and in a cylindrical surface for the rail [33].

$\mathbf{F}_{J i}^{1 V}, \mathbf{F}_{J i}^{1 C}, \mathbf{F}_{j i}^{1 A}, \mathbf{F}_{J i}^{2 V}, \mathbf{F}_{J i}^{2 C}, \mathbf{F}_{J i}^{2 A}$, and $\mathbf{F}_{J i}^{2 G}$ for the $i$ th motor car can be obtained similarly.

The load vector of the rail $\mathbf{F}_{r}$ of order $2 \bar{N}_{r} \times 1$ can be written as

$$
\begin{aligned}
\mathbf{F}_{r}= & {\left[\begin{array}{c}
\mathbf{F}_{r}^{L} \\
\mathbf{F}_{r}^{R}
\end{array}\right], } \\
\mathbf{F}_{r}^{L}= & \mathbf{F}_{r}^{L 0}+\mathbf{F}_{r}^{L 1}+\mathbf{F}_{r}^{L 2}+\mathbf{F}_{r}^{L 3}+\mathbf{F}_{r}^{L 4}+\mathbf{F}_{r}^{L 5}+\mathbf{F}_{r}^{L 6}+\mathbf{F}_{r}^{L 7} \\
& +\mathbf{F}_{r}^{L 8}+\mathbf{F}_{r}^{L 9}+\mathbf{F}_{r}^{L 10}, \\
\mathbf{F}_{r}^{R}= & \mathbf{F}_{r}^{R 0}+\mathbf{F}_{r}^{R 1}+\mathbf{F}_{r}^{R 2}+\mathbf{F}_{r}^{R 3}+\mathbf{F}_{r}^{R 4}+\mathbf{F}_{r}^{R 5}+\mathbf{F}_{r}^{R 6}+\mathbf{F}_{r}^{R 7} \\
& +\mathbf{F}_{r}^{R 8}+\mathbf{F}_{r}^{R 9}+\mathbf{F}_{r}^{R 10},
\end{aligned}
$$

where $\mathbf{F}_{r}^{L 0}, \mathbf{F}_{r}^{L 1}, \mathbf{F}_{r}^{L 2}, \mathbf{F}_{r}^{L 3}$, and $\mathbf{F}_{r}^{L 4}$ represent the load vectors of each wheelset acting upon the left rail caused by the train's weight, the track elevation irregularity, the cross level irregularity, the alignment irregularity, and the gauge irregularity, respectively; $\mathbf{F}_{r}^{L 5}, \mathbf{F}_{r}^{L 6}, \mathbf{F}_{r}^{L 7}$, and $\mathbf{F}_{r}^{L 8}$ represent the load vectors of each wheelset acting upon the left rail caused by the velocity of track elevation, cross level, alignment, and gauge irregularities, respectively; $\mathbf{F}_{r}^{L 9}$ and $\mathbf{F}_{r}^{L 10}$ represent the load vectors of each wheelset acting upon the left rail caused by the acceleration of track elevation and cross level irregularities, respectively. Similarly, $\mathbf{F}_{r}^{R 0}-\mathbf{F}_{r}^{R 10}$ represent the load vectors of each wheelset acting upon the right rail.

$\mathbf{F}_{r}^{L 0}-\mathbf{F}_{r}^{L 10}$ can be written, respectively, as

$$
\begin{aligned}
\mathbf{F}_{r}^{L 0}= & -\sum_{j=1}^{N_{v}} \sum_{h=1}^{4} \frac{1}{2} g\left(m_{w}+\frac{1}{2} m_{t}+\frac{1}{4} m_{c}\right) \mathbf{N}_{z j h}^{\mathbf{T}} \\
& -\sum_{i=1}^{2} \sum_{h=1}^{4} \frac{1}{2} g\left(m_{J w}+\frac{1}{2} m_{J t}+\frac{1}{4} m_{J c}\right) \mathbf{N}_{z J i h}^{\mathbf{T}}, \\
\mathbf{F}_{r}^{L 1}= & -\sum_{j=1}^{N_{v}} \sum_{h=1}^{4} k_{p z} r\left(x_{j h}^{V}\right) \mathbf{N}_{z j h}^{\mathbf{T}} \\
& -\sum_{i=1}^{2} \sum_{h=1}^{4} k_{J p z} r\left(x_{J i h}^{V}\right) \mathbf{N}_{z J i h}^{\mathbf{T}}, \\
\mathbf{F}_{r}^{L 2}= & -\sum_{j=1}^{N_{v}} \sum_{h=1}^{4} \frac{1}{2} k_{p z} r\left(x_{j h}^{C}\right) \mathbf{N}_{z j h}^{\mathbf{T}} \\
& -\sum_{i=1}^{2} \sum_{h=1}^{4} \frac{1}{2} k_{J p z} r\left(x_{J i h}^{C}\right) \mathbf{N}_{z J i h}^{\mathbf{T}}, \\
& -\sum_{i=1}^{N_{v}} \sum_{h=1}^{4} \frac{W_{J a x l e} \lambda}{b_{0}} r\left(x_{J i h}^{A}\right) \mathbf{N}_{z J i h}^{\mathbf{T}}, \\
\mathbf{F}_{r}^{L 3}= & -\sum_{h=1} \frac{W_{0 x l e}}{b_{0}} r\left(x_{j h}^{A}\right) \mathbf{N}_{z j h}^{\mathbf{T}} \\
&
\end{aligned}
$$

$$
\begin{aligned}
\mathbf{F}_{r}^{L 4}= & -\sum_{j=1}^{N_{v}} \sum_{h=1}^{4} \frac{W_{\mathrm{axle}} \lambda}{2 b_{0}} r\left(x_{j h}^{G}\right) \mathbf{N}_{z j h}^{\mathbf{T}} \\
& -\sum_{i=1}^{2} \sum_{h=1}^{4} \frac{W_{\text {Jaxle }} \lambda}{2 b_{0}} r\left(x_{J i h}^{G}\right) \mathbf{N}_{z J i h}^{\mathbf{T}},
\end{aligned}
$$$$
\mathbf{F}_{r}^{L 5}=-\sum_{j=1}^{N_{v}} \sum_{h=1}^{4} c_{p z} \dot{r}\left(x_{j h}^{V}\right) \mathbf{N}_{z j h}^{\mathbf{T}}
$$$$
-\sum_{i=1}^{2} \sum_{h=1}^{4} c_{J p z} \dot{r}\left(x_{J i h}^{V}\right) \mathbf{N}_{z J i h}^{\mathrm{T}},
$$$$
\mathbf{F}_{r}^{L 6}=-\sum_{j=1}^{N_{v}} \sum_{h=1}^{4} \frac{1}{2} c_{p z} \dot{r}\left(x_{j h}^{C}\right) \mathbf{N}_{z j h}^{\mathbf{T}}
$$$$
-\sum_{i=1}^{2} \sum_{h=1}^{4} \frac{1}{2} c_{J p z} \dot{r}\left(x_{J i h}^{C}\right) \mathbf{N}_{z J i h}^{\mathbf{T}},
$$$$
\mathbf{F}_{r}^{L 7}=-\sum_{j=1}^{N_{v}} \sum_{h=1}^{4} f_{L j h}^{22} \dot{r}\left(x_{j h}^{A}\right) \mathbf{N}_{y j h}^{\mathbf{T}}
$$$$
-\sum_{i=1}^{2} \sum_{h=1}^{4} f_{L J i h}^{22} \dot{r}\left(x_{J i h}^{A}\right) \mathbf{N}_{y J i h}^{\mathrm{T}},
$$$$
\mathbf{F}_{r}^{L 8}=-\sum_{j=1}^{N_{v}} \sum_{h=1}^{4} \frac{1}{2} f_{L j h}^{22} \dot{r}\left(x_{j h}^{G}\right) \mathbf{N}_{y j h}^{\mathbf{T}}
$$$$
-\sum_{i=1}^{2} \sum_{h=1}^{4} \frac{1}{2} f_{L J i h}^{22} \dot{r}\left(x_{J i h}^{G}\right) \mathbf{N}_{y J i h}^{\mathbf{T}},
$$$$
\mathbf{F}_{r}^{L 9}=-\sum_{j=1}^{N_{v}} \sum_{h=1}^{4} \frac{1}{2} m_{w} \ddot{r}\left(x_{j h}^{V}\right) \mathbf{N}_{z j h}^{\mathbf{T}}
$$$$
-\sum_{i=1}^{2} \sum_{h=1}^{4} \frac{1}{2} m_{J w} \ddot{r}\left(x_{J i h}^{V}\right) \mathbf{N}_{z J i h}^{\mathrm{T}},
$$$$
\mathbf{F}_{r}^{L 10}=-\sum_{j=1}^{N_{v}} \sum_{h=1}^{4} \frac{1}{4} m_{w} \ddot{r}\left(x_{j h}^{C}\right) \mathbf{N}_{z j h}^{\mathrm{T}}
$$$$
-\sum_{i=1}^{2} \sum_{h=1}^{4} \frac{1}{4} m_{J w} \ddot{r}\left(x_{J i h}^{C}\right) \mathbf{N}_{z J i h}^{\mathrm{T}},
$$

where $\mathbf{N}_{z j h}$ and $\mathbf{N}_{z J i h}$, both of order $1 \times \bar{N}_{r}$, are the timedependent shape function vectors in the $x z$ plane for the rail element evaluated at the position of the $h$ th wheelset of the $j$ th trailer car and the $i$ th motor car, respectively; $\mathbf{N}_{y j h}$ and $\mathbf{N}_{y J i h}$, both of order $1 \times \bar{N}_{r}$, are the time-dependent shape function vectors in the $x y$ plane for the rail element evaluated at the position of the $h$ th wheelset of the $j$ th trailer car and the $i$ th motor car, respectively; $g$ is the acceleration of gravity; $W_{J \text { axle }}$ is the axle weight of the motor car; $\ddot{r}(\cdot)$ is the second derivative 
of track irregularity; and $f_{L J i h}^{22}(h=1-4)$ is the lateral creepage coefficient between the $h$ th wheelset of the $i$ th motor car and the left rail.

$\mathbf{F}_{r}^{R 0}-\mathbf{F}_{r}^{R 10}$ can be derived similarly.

Each element for the load vector of the slab $\mathbf{F}_{s}$ of order $\bar{N}_{s} \times 1$ is zero.

The load vector of the girder $\mathbf{F}_{b}$ of order $\bar{N}_{b} \times 1$ can be written as

$$
\begin{aligned}
\mathbf{F}_{b} & =\left[\begin{array}{llll}
\mathbf{F}_{b 1} & \mathbf{F}_{b 2} & \cdots & \mathbf{F}_{b N_{b}}
\end{array}\right]^{\mathbf{T}}, \\
\mathbf{F}_{b i} & =\left[\begin{array}{lllll}
\mathbf{f}_{b 1} & \mathbf{f}_{b 2} & \cdots & \mathbf{f}_{b n_{b i}} & \mathbf{f}_{b n_{b i}+1}
\end{array}\right], \\
\mathbf{f}_{b i} & =\left[\begin{array}{lllll}
m_{b i} a_{g y} & m_{b i} a_{g z} & 0 & 0 & 0
\end{array}\right],
\end{aligned}
$$

where $n_{b i}$ denotes the number of elements of the $i$ th girder, $m_{b i}$ the mass of node of the $i$ th girder, and $a_{y g}$ and $a_{z g}$ the lateral horizontal and vertical seismic accelerations, respectively.

Similarly the load vector of the pier $\mathbf{F}_{p}$ of order $\bar{N}_{p} \times 1$ can be written as

$$
\begin{aligned}
& \mathbf{F}_{p}=\left[\begin{array}{llll}
\mathbf{F}_{p 1} & \mathbf{F}_{p 2} & \cdots & \mathbf{F}_{p N_{p}}
\end{array}\right]^{\mathbf{T}}, \\
& \mathbf{F}_{p i}=\left[\begin{array}{lllll}
\mathbf{f}_{p 1} & \mathbf{f}_{p 2} & \cdots & \mathbf{f}_{p n_{p i}} & \mathbf{f}_{p n_{p i}+1}
\end{array}\right], \\
& \mathbf{f}_{p i}=\left[\begin{array}{lll}
m_{p i} a_{g y} & m_{p i} a_{g z} & 0
\end{array}\right],
\end{aligned}
$$

where $n_{p i}$ denotes the number of element of the $i$ th pier and $m_{p i}$ the mass of node of the $i$ th pier.

Let the following definitions be true:

$$
\begin{aligned}
& \mathbf{F}_{\mathrm{GRT}}(t)=\left[\begin{array}{c}
\mathbf{0}_{T_{\mathrm{dof}} \times 1} \\
\mathbf{F}_{r}^{L 0} \\
\mathbf{F}_{r}^{R 0} \\
\mathbf{0}_{\bar{N}_{s} \times 1} \\
\mathbf{0}_{\bar{N}_{b} \times 1} \\
\mathbf{0}_{\bar{N}_{p} \times 1}
\end{array}\right], \\
& \mathbf{F}_{\mathrm{RND}}^{1}(t)=\left[\begin{array}{c}
\mathbf{F}_{t}^{1 V} \\
\mathbf{F}_{r}^{L 1} \\
\mathbf{F}_{r}^{R 1} \\
\mathbf{0}_{\bar{N}_{s} \times 1}^{1 V} \\
\mathbf{0}_{\bar{N}_{b} \times 1} \\
\mathbf{0}_{\bar{N}_{p} \times 1}
\end{array}\right], \\
& \mathbf{F}_{\mathrm{RND}}^{2}(t)=\left[\begin{array}{c}
\mathbf{F}_{t}^{1 C} \\
\mathbf{F}_{r}^{L 2} \\
\mathbf{F}_{r}^{R 2} \\
\mathbf{0}_{\bar{N}_{s} \times 1}^{2} \\
\mathbf{0}_{\bar{N}_{b} \times 1} \\
\mathbf{0}_{\bar{N}_{p} \times 1}
\end{array}\right],
\end{aligned}
$$$$
\mathbf{F}_{\mathrm{RND}}^{3}(t)=\left[\begin{array}{c}
\mathbf{F}_{t}^{1 A} \\
\mathbf{F}_{r}^{L 3} \\
\mathbf{F}_{r}^{R 3} \\
\mathbf{0}_{\bar{N}_{s} \times 1}^{2} \\
\mathbf{0}_{\bar{N}_{b} \times 1} \\
\mathbf{0}_{\bar{N}_{p} \times 1}
\end{array}\right],
$$$$
\mathbf{F}_{\mathrm{RND}}^{4}(t)=\left[\begin{array}{c}
\mathbf{0}_{T_{\mathrm{dof}} \times 1} \\
\mathbf{F}_{r}^{L 4} \\
\mathbf{F}_{r}^{R 4} \\
\mathbf{0}_{\bar{N}_{s} \times 1} \\
\mathbf{0}_{\bar{N}_{b} \times 1} \\
\mathbf{0}_{\bar{N}_{p} \times 1}
\end{array}\right],
$$$$
\mathbf{F}_{\mathrm{RND}}^{5}(t)=\left[\begin{array}{c}
\mathbf{F}_{t}^{2 V} \\
\mathbf{F}_{r}^{L 5} \\
\mathbf{F}_{r}^{R 5} \\
\mathbf{0}_{\bar{N}_{s} \times 1} \\
\mathbf{0}_{\bar{N}_{b} \times 1} \\
\mathbf{0}_{\bar{N}_{p} \times 1}
\end{array}\right],
$$$$
\mathbf{F}_{\mathrm{RND}}^{6}(t)=\left[\begin{array}{c}
\mathbf{F}_{t}^{2 C} \\
\mathbf{F}_{r}^{L 6} \\
\mathbf{F}_{r}^{R 6} \\
\mathbf{0}_{\bar{N}_{s} \times 1} \\
\mathbf{0}_{\bar{N}_{b} \times 1} \\
\mathbf{0}_{\bar{N}_{p} \times 1}
\end{array}\right],
$$$$
\mathbf{F}_{\mathrm{RND}}^{7}(t)=\left[\begin{array}{c}
\mathbf{F}_{t}^{2 A} \\
\mathbf{F}_{r}^{L 7} \\
\mathbf{F}_{r}^{R 7} \\
\mathbf{0}_{\bar{N}_{s} \times 1} \\
\mathbf{0}_{\bar{N}_{b} \times 1} \\
\mathbf{0}_{\bar{N}_{p} \times 1}
\end{array}\right],
$$$$
\mathbf{F}_{\mathrm{RND}}^{8}(t)=\left[\begin{array}{c}
\mathbf{F}_{t}^{2 G} \\
\mathbf{F}_{r}^{L 8} \\
\mathbf{F}_{r}^{R 8} \\
\mathbf{0}_{\bar{N}_{s} \times 1} \\
\mathbf{0}_{\bar{N}_{b} \times 1} \\
\mathbf{0}_{\bar{N}_{p} \times 1}
\end{array}\right],
$$ 


$$
\begin{aligned}
& \mathbf{F}_{\mathrm{RND}}^{9}(t)=\left[\begin{array}{c}
\mathbf{0}_{T_{\mathrm{dof}} \times 1} \\
\mathbf{F}_{r}^{L 9} \\
\mathbf{F}_{r}^{R 9} \\
\mathbf{0}_{\bar{N}_{s} \times 1} \\
\mathbf{0}_{\bar{N}_{b} \times 1} \\
\mathbf{0}_{\bar{N}_{p} \times 1}
\end{array}\right], \\
& \mathbf{F}_{\mathrm{RND}}^{10}(t)=\left[\begin{array}{c}
\mathbf{0}_{T_{\mathrm{dof}} \times 1} \\
\mathbf{F}_{r}^{L 10} \\
\mathbf{F}_{r}^{R 10} \\
\mathbf{0}_{\bar{N}_{s} \times 1} \\
\mathbf{0}_{\bar{N}_{b} \times 1} \\
\mathbf{0}_{\bar{N}_{p} \times 1}
\end{array}\right], \\
& \mathbf{F}_{\mathrm{RND}}^{11}(t)=\left[\begin{array}{c}
\mathbf{0}_{T_{\mathrm{dof}} \times 1} \\
\mathbf{0}_{N_{r} \times 1} \\
\mathbf{0}_{N_{r} \times 1} \\
\mathbf{0}_{\bar{N}_{s} \times 1} \\
\mathbf{F}_{b} \\
\mathbf{F}_{p}
\end{array}\right] .
\end{aligned}
$$

Then, the load vector of the TSTBI system simultaneously excited by track irregularities and earthquakes $\mathbf{F}(t)$ can be expressed as

$$
\mathbf{F}(t)=\left[\begin{array}{c}
\mathbf{F}_{t} \\
\mathbf{F}_{r}^{L} \\
\mathbf{F}_{r}^{R} \\
\mathbf{F}_{s} \\
\mathbf{F}_{b} \\
\mathbf{F}_{p}
\end{array}\right]=\mathbf{F}_{\mathrm{GRT}}(t)+\sum_{i=1}^{11} \mathbf{F}_{\mathrm{RND}}^{i}(t) .
$$

\section{Random Vibration Analysis of the TSTBI System under the Actions of Track Irregularities and Earthquakes by PEM}

4.1. Pseudoexcitation for the System. PEM is a highly efficient and accurate algorithm for random vibration analysis, which has been established and successfully used for many timedependent systems subjected to different kinds of random excitations, for example, earthquakes [27] and wind gusts [34].

For any zero-mean-valued random system, the relationship between the PSD $\boldsymbol{S}_{F F}$ of excitation $\mathbf{F}_{\mathrm{RND}}$ and the PSD $\mathbf{S}_{U U}$ of response $\mathbf{U}$ can be expressed as

$$
\mathbf{S}_{U U}=\mathbf{H}^{2}(\omega) \mathbf{S}_{F F},
$$

where $\mathbf{H}(\omega)$ denotes the frequency response function.
The basic principle of PEM is the following. Given the PSD function $\boldsymbol{S}_{F F}$ and defining the pseudoexcitation $\widetilde{\mathbf{F}}_{\mathrm{RND}}=$ $\sqrt{\mathbf{S}_{F F}} e^{i \omega t}$, one then can obtain, according to (24),

$$
\widetilde{\mathbf{U}}=\sqrt{\mathbf{S}_{F F}} \mathbf{H}(\omega) e^{i \omega t}
$$

with

$$
\begin{aligned}
\widetilde{\mathbf{F}}_{\mathrm{RND}}^{*} \widetilde{\mathbf{F}}_{\mathrm{RND}} & =\sqrt{\mathbf{S}_{F F}} e^{-i \omega t} \sqrt{\mathbf{S}_{F F}} e^{i \omega t}=\mathbf{S}_{F F}, \\
\widetilde{\mathbf{U}}^{*} \widetilde{\mathbf{U}} & =\sqrt{\mathbf{S}_{U U}} \mathbf{H}^{*}(\omega) e^{-i \omega t} \sqrt{\mathbf{S}_{U U}} \mathbf{H}(\omega) e^{i \omega t} \\
& =\mathbf{H}^{*}(\omega) \mathbf{S}_{F F} \mathbf{H}(\omega)=\mathbf{S}_{U U},
\end{aligned}
$$

where $\widetilde{\mathrm{U}}$ denotes the pseudoresponse and the superscript " $*$ " the complex conjugate.

Let $x$ be the distance from the left-hand starting point of the TSTBI model to the wheel-rail contact point at time $t$. There then exists a transformation from the PSD $\mathbf{S}_{r r}(\Omega)$ of $r(x)$ in the space domain to the PSD $\mathbf{S}_{r r}(\omega)$ of $r(t)$ in the time domain, according to $x=v t$ :

$$
\mathbf{S}_{r r}(\omega)=\frac{\mathbf{S}_{r r}(\Omega)}{v},
$$

where $\omega(\mathrm{rad} / \mathrm{s})$ denotes the time frequency, $\Omega(\mathrm{rad} / \mathrm{m})$ the spatial frequency, and $v(\mathrm{~m} / \mathrm{s})$ the train speed. Obviously, $\omega=$ $\Omega v$.

As the duration of an earthquake is usually comparable with the time it takes for a train to pass over a large bridge, it is reasonable to represent the earthquakes by the nonstationary random process as follows:

$$
\begin{aligned}
& a_{g y}=\mathbf{g}(t) a_{y}, \\
& a_{g z}=\mathbf{g}(t) a_{z},
\end{aligned}
$$

where $a_{y}$ and $a_{z}$ denote the zero-mean-valued, stationary, lateral, and vertical seismic accelerations with the PSDs of $\mathbf{S}_{a_{y} a_{y}}(\omega)$ and $\mathbf{S}_{a_{z} a_{z}}(\omega)$, respectively, and $\mathbf{g}(t)$ a specified slowly varying modulation function.

According to PEM and (23), the pseudoexcitation $\widetilde{\mathbf{F}}_{\mathrm{RND}}(\omega, t)$ induced by $\mathbf{F}_{\mathrm{RND}}(t)$ can be written as

$$
\widetilde{\mathbf{F}}_{\mathrm{RND}}(\omega, t)=\sum_{i=1}^{11} \widetilde{\mathbf{F}}_{\mathrm{RND}}^{i}(\omega, t)
$$

with

$$
\widetilde{\mathbf{F}}_{\mathrm{RND}}^{1}(\omega, t)=\left[\begin{array}{c}
\widetilde{\mathbf{F}}_{t}^{1 V}(\omega, t) \\
\widetilde{\mathbf{F}}_{r}^{L 1}(\omega, t) \\
\widetilde{\mathbf{F}}_{r}^{R 1}(\omega, t) \\
\mathbf{0}_{\bar{N}_{s} \times 1} \\
\mathbf{0}_{\bar{N}_{b} \times 1} \\
\mathbf{0}_{\bar{N}_{p} \times 1}
\end{array}\right],
$$


$\widetilde{\mathbf{F}}_{\mathrm{RND}}^{2}(\omega, t)=\left[\begin{array}{c}\widetilde{\mathbf{F}}_{t}^{1 C}(\omega, t) \\ \widetilde{\mathbf{F}}_{r}^{L 2}(\omega, t) \\ \widetilde{\mathbf{F}}_{r}^{R 2}(\omega, t) \\ \mathbf{0}_{\bar{N}_{s} \times 1} \\ \mathbf{0}_{\bar{N}_{b} \times 1} \\ \mathbf{0}_{\bar{N}_{p} \times 1}\end{array}\right]$,

$\widetilde{\mathbf{F}}_{\mathrm{RND}}^{3}(\omega, t)=\left[\begin{array}{c}\widetilde{\mathbf{F}}_{t}^{1 A}(\omega, t) \\ \widetilde{\mathbf{F}}_{r}^{L 3}(\omega, t) \\ \widetilde{\mathbf{F}}_{r}^{R 3}(\omega, t) \\ \mathbf{0}_{\bar{N}_{s} \times 1} \\ \mathbf{0}_{\bar{N}_{b} \times 1} \\ \mathbf{0}_{\bar{N}_{p} \times 1}\end{array}\right]$,

$\widetilde{\mathbf{F}}_{\mathrm{RND}}^{4}(\omega, t)=\left[\begin{array}{c}\mathbf{0}_{T_{\mathrm{dof}} \times 1} \\ \widetilde{\mathbf{F}}_{r}^{L 4}(\omega, t) \\ \widetilde{\mathbf{F}}_{r}^{R 4}(\omega, t) \\ \mathbf{0}_{\bar{N}_{s} \times 1} \\ \mathbf{0}_{\bar{N}_{b} \times 1} \\ \mathbf{0}_{\bar{N}_{p} \times 1}\end{array}\right]$,

$\widetilde{\mathbf{F}}_{\mathrm{RND}}^{5}(\omega, t)=\left[\begin{array}{c}\widetilde{\mathbf{F}}_{t}^{2 V}(\omega, t) \\ \widetilde{\mathbf{F}}_{r}^{L 5}(\omega, t) \\ \widetilde{\mathbf{F}}_{r}^{R 5}(\omega, t) \\ \mathbf{0}_{\bar{N}_{s} \times 1} \\ \mathbf{0}_{\bar{N}_{b} \times 1} \\ \mathbf{0}_{\bar{N}_{p} \times 1}\end{array}\right]$,

$\widetilde{\mathbf{F}}_{\mathrm{RND}}^{6}(\omega, t)=\left[\begin{array}{c}\widetilde{\mathbf{F}}_{t}^{2 C}(\omega, t) \\ \widetilde{\mathbf{F}}_{r}^{L 6}(\omega, t) \\ \widetilde{\mathbf{F}}_{r}^{R 6}(\omega, t) \\ \mathbf{0}_{\bar{N}_{s} \times 1} \\ \mathbf{0}_{\bar{N}_{b} \times 1} \\ \mathbf{0}_{\bar{N}_{p} \times 1}\end{array}\right]$,

$\widetilde{\mathbf{F}}_{\mathrm{RND}}^{7}(\omega, t)=\left[\begin{array}{c}\widetilde{\mathbf{F}}_{t}^{2 A}(\omega, t) \\ \widetilde{\mathbf{F}}_{r}^{L 7}(\omega, t) \\ \widetilde{\mathbf{F}}_{r}^{R 7}(\omega, t) \\ \mathbf{0}_{\bar{N}_{s} \times 1} \\ \mathbf{0}_{\bar{N}_{b} \times 1} \\ \mathbf{0}_{\bar{N}_{p} \times 1}\end{array}\right]$,
$\widetilde{\mathbf{F}}_{\mathrm{RND}}^{8}(\omega, t)=\left[\begin{array}{c}\widetilde{\mathbf{F}}_{t}^{2 G}(\omega, t) \\ \widetilde{\mathbf{F}}_{r}^{L 8}(\omega, t) \\ \widetilde{\mathbf{F}}_{r}^{R 8}(\omega, t) \\ \mathbf{0}_{\bar{N}_{s} \times 1} \\ \mathbf{0}_{\bar{N}_{b} \times 1} \\ \mathbf{0}_{\bar{N}_{p} \times 1}\end{array}\right]$,

$\widetilde{\mathbf{F}}_{\mathrm{RND}}^{9}(\omega, t)=\left[\begin{array}{c}\mathbf{0}_{T_{\mathrm{dof}} \times 1} \\ \widetilde{\mathbf{F}}_{r}^{L 9}(\omega, t) \\ \widetilde{\mathbf{F}}_{r}^{R 9}(\omega, t) \\ \mathbf{0}_{\bar{N}_{s} \times 1} \\ \mathbf{0}_{\bar{N}_{b} \times 1} \\ \mathbf{0}_{\bar{N}_{p} \times 1}\end{array}\right]$,

$\widetilde{\mathbf{F}}_{\mathrm{RND}}^{10}(\omega, t)=\left[\begin{array}{c}\mathbf{0}_{T_{\mathrm{dof}} \times 1} \\ \widetilde{\mathbf{F}}_{r}^{L 10}(\omega, t) \\ \widetilde{\mathbf{F}}_{r}^{R 10}(\omega, t) \\ \mathbf{0}_{\bar{N}_{s} \times 1} \\ \mathbf{0}_{\bar{N}_{b} \times 1} \\ \mathbf{0}_{\bar{N}_{p} \times 1}\end{array}\right]$,

$\widetilde{\mathbf{F}}_{\mathrm{RND}}^{11}(\omega, t)=\left[\begin{array}{c}\mathbf{0}_{T_{\mathrm{dof}} \times 1} \\ \mathbf{0}_{N_{r} \times 1} \\ \mathbf{0}_{N_{r} \times 1} \\ \mathbf{0}_{\bar{N}_{s} \times 1} \\ \widetilde{\mathbf{F}}_{b}(\omega, t) \\ \widetilde{\mathbf{F}}_{p}(\omega, t)\end{array}\right]$.

4.2. Calculation of Random Responses of the System. Based on (1) and (23), the equations of motion for the TSTBI system can be expressed as

$$
\mathbf{M}(t) \ddot{\mathbf{U}}+\mathbf{C}(t) \dot{\mathbf{U}}+\mathbf{K}(t) \mathbf{U}=\mathbf{F}_{\mathrm{GRT}}(t)+\mathbf{F}_{\mathrm{RND}}(t),
$$

where $\mathbf{M}(t), \mathbf{C}(t)$, and $\mathbf{K}(t)$ denote the mass, damping, and stiffness matrices, respectively, of the total TSTBI system; $\mathbf{U}$, $\dot{\mathbf{U}}$, and $\ddot{\mathbf{U}}$ denote the displacement, velocity, and acceleration vectors, respectively, of the system.

The response of the system caused by the train's weight $\mathbf{F}_{\mathrm{GRT}}(t)$ is deterministic and can be obtained easily in the time domain using a step-by-step integration method. While, the pseudoresponse $\widetilde{\mathbf{U}}(\omega, t)$ of the system caused by the pseudoexcitation $\widetilde{\mathbf{F}}_{\mathrm{RND}}(\omega, t)$ can be obtained by solving the following equation in both the frequency and time domains:

$$
\mathbf{M}(t) \widetilde{\ddot{\mathbf{U}}}+\mathbf{C}(t) \widetilde{\dot{\mathbf{U}}}+\mathbf{K}(t) \widetilde{\mathbf{U}}=\widetilde{\mathbf{F}}_{\mathrm{RND}}(\omega, t)
$$


According to PEM, the time-dependent $\operatorname{PSD~}_{U U}(\omega, t)$ of arbitrary response $\widetilde{\mathbf{U}}(\omega, t)$ of the system can be written as

$$
\mathbf{S}_{U U}(\omega, t)=\widetilde{\mathbf{U}}^{*}(\omega, t) \widetilde{\mathbf{U}}^{\mathrm{T}}(\omega, t) .
$$

Then, one can estimate the global maximum value $\max (\mathbf{U}(t))$, the minimum value $\min (\mathbf{U}(t))$ and the dynamic reliability $p$ of the random responses by the first-passage failure criterion according to the following equations $[6,35$, 36]:

$$
\begin{aligned}
\max (\mathbf{U}(t)) & =\max (\overline{\mathbf{U}}(t))+\left(E(\eta)+\kappa \sigma_{\eta}\right) \bar{\sigma}_{\mathbf{U}}, \\
\min (\mathbf{U}(t)) & =\min (\overline{\mathbf{U}}(t))-\left(E(\eta)+\kappa \sigma_{\eta}\right) \bar{\sigma}_{\mathbf{U}}, \\
p(b,-b) & =\exp \left\{\int_{0}^{T}-v(t) \exp \left(\frac{-b^{2}}{2 \sigma_{\mathbf{U}}^{2}(t)}\right)\right\},
\end{aligned}
$$

where $\eta$ is a dimensionless parameter known as the peak factor, $\kappa$ is a safety factor, $E(\eta)$ and $\sigma_{\eta}$ are the expectation value and the standard deviation of $\eta$, respectively, $\bar{\sigma}_{\mathrm{U}}$ is the equivalent stationary standard deviation of random response $\mathbf{U}, v$ is the zero-passage rate, and $b$ is the allowable safety limit.

4.3. Solution Procedures. The flowchart for efficiently analyzing the random vibrations of the TSTBI system under the actions of track irregularities and seismic accelerations is shown in Figure 2.

\section{Case Study}

5.1. Properties of the TSTBI System. A fifteen-span simply supported boxing girder high-speed railway bridge, with the span length of $32 \mathrm{~m}$ and the pier height of $20 \mathrm{~m}$, is considered as shown in Figure 1. The central part of the slab track is supported on the bridge, while the left and right parts of the track are supported on the subgrades adjacent to the bridge. The lengths of element of the rail, slab, and girder are all equal to the fastener spacing of $0.625 \mathrm{~m}$, while the length of the pier element equals $1.0 \mathrm{~m}$. The high-speed train comprises one front motor car, six trailer cars, and one rear motor car moving at a constant velocity $v$. The fundamental data of the motor car and trailer car can refer to [37]. The major fundamental data of the slab track and bridge are listed in Table 1. The PSDs of the German high-speed track spectrum of low irregularity [38] are adopted. In addition, the PSDs of the lateral horizontal and vertical seismic accelerations and the uniform modulation function (Figure 3 ) are taken as follows $[6,35]$ :

$$
\begin{aligned}
\mathbf{S}_{a_{y} a_{y}}(\omega)= & \frac{\omega_{g y}^{4}+4 \zeta_{g y}^{2} \omega_{g y}^{2} \omega^{2}}{\left(\omega_{g y}^{2}-\omega^{2}\right)^{2}+4 \zeta_{g y}^{2} \omega_{g y}^{2} \omega^{2}} \\
& \cdot \frac{\omega^{4}}{\left(\omega^{2}-\omega_{f y}^{2}\right)^{2}+4 \zeta_{f y}^{2} \omega_{f y}^{2} \omega^{2}} \mathbf{S}_{0 y},
\end{aligned}
$$

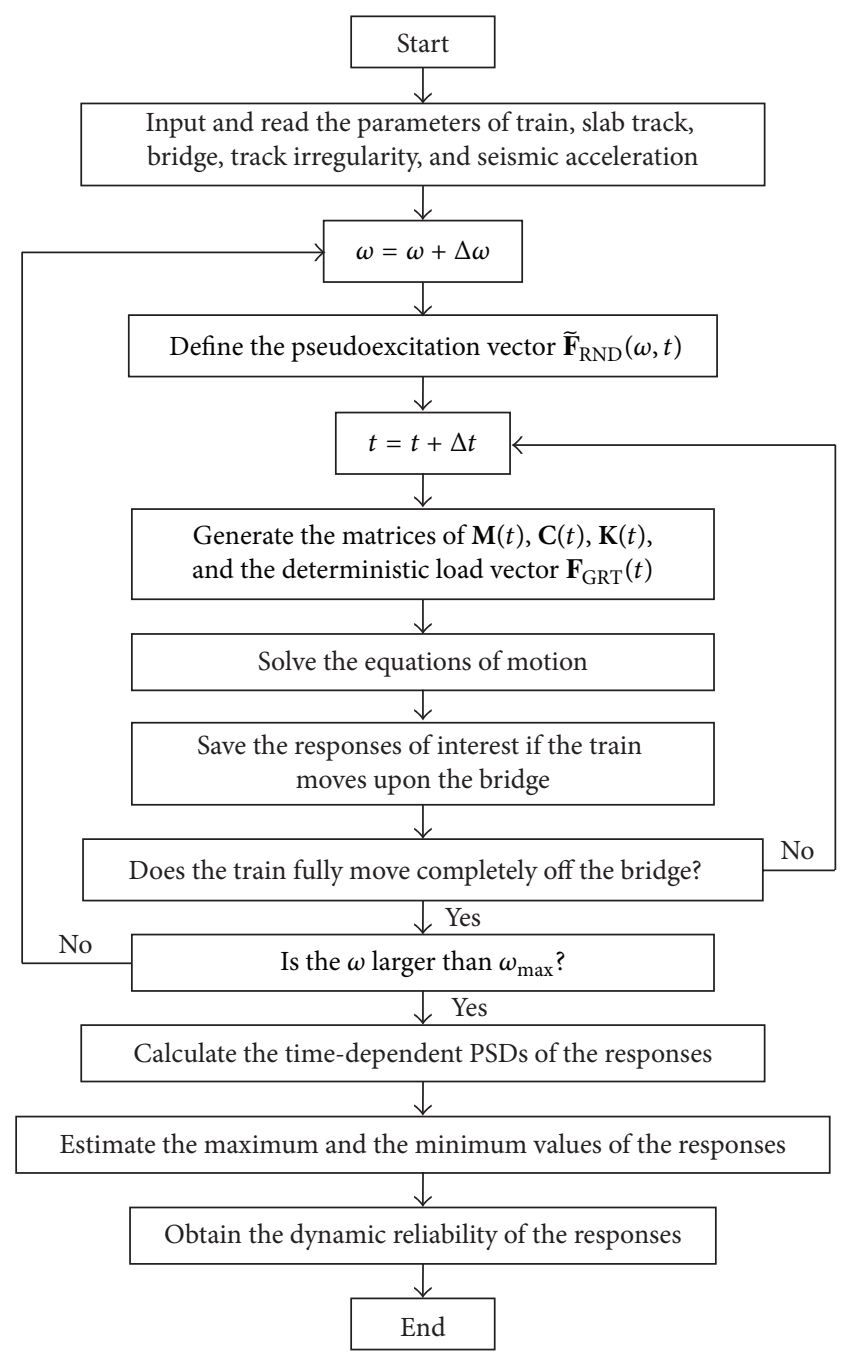

FIGURE 2: Flowchart for analyzing the random vibrations of the TSTBI system.

$$
\begin{aligned}
\mathbf{S}_{a_{z} a_{z}}(\omega)= & \frac{\omega_{g z}^{4}+4 \zeta_{g z}^{2} \omega_{g z}^{2} \omega^{2}}{\left(\omega_{g z}^{2}-\omega^{2}\right)^{2}+4 \zeta_{g z}^{2} \omega_{g z}^{2} \omega^{2}} \\
& \cdot \frac{\omega^{4}}{\left(\omega^{2}-\omega_{f z}^{2}\right)^{2}+4 \zeta_{f z}^{2} \omega_{f z}^{2} \omega^{2}} \mathbf{S}_{0 z}, \\
\mathbf{g}(t)= & \begin{cases}\left(\frac{t}{t_{b}}\right)^{2} & 0 \leq t<t_{b} \\
1 & t_{b} \leq t<t_{c} \\
\exp \left(c\left(t-t_{c}\right)\right) & t \geq t_{c},\end{cases}
\end{aligned}
$$

where $\mathbf{S}_{0 y}$ and $\mathbf{S}_{0 z}$ denote the spectral intensity factor, $\zeta_{g y}$ and $\zeta_{g z}$ denote the damping ratio of the site, $\omega_{g y}$ and $\omega_{g z}$ denote the predominant frequency of the site, $\zeta_{f y}, \zeta_{f z}, \omega_{f y}$, and $\omega_{f z}$ denote the filter parameters, $c$ denotes the attenuation coefficient, and $t_{b}$ and $t_{c}$ denote the times at the start and end, respectively, of the stationary main shock. Additionally, $\zeta_{g z}=\zeta_{g y}, \omega_{g z}=1.58 \omega_{g y}, \zeta_{f y}=\zeta_{g y}, \omega_{f y}=0.1 \omega_{g y}-0.2 \omega_{g y}$, 
TABLE 1: Major parameters for slab track and bridge.

\begin{tabular}{|c|c|c|c|}
\hline Notation & Item & Unit & Value \\
\hline \multicolumn{4}{|l|}{ Slab track } \\
\hline$E_{r}$ & Young's modulus of rail & $\mathrm{N} / \mathrm{m}^{2}$ & $2.06 \times 10^{11}$ \\
\hline$I_{r y}$ & Flexural moment of inertia about $y$-axis of cross section of rail & $\mathrm{m}^{4}$ & $3.217 \times 10^{-5}$ \\
\hline$I_{r z}$ & Flexural moment of inertia about $z$-axis of cross section of rail & $\mathrm{m}^{4}$ & $5.24 \times 10^{-6}$ \\
\hline $\bar{m}_{r}$ & Mass per unit length of rail & $\mathrm{kg} / \mathrm{m}$ & 60.64 \\
\hline$E_{s}$ & Young's modulus of slab & $\mathrm{N} / \mathrm{m}^{2}$ & $3.6 \times 10^{10}$ \\
\hline$I_{s y}$ & Flexural moment of inertia of cross section of slab & $\mathrm{m}^{4}$ & $1.4 \times 10^{-3}$ \\
\hline$I_{s z}$ & Flexural moment of inertia of cross section of slab & $\mathrm{m}^{4}$ & 0.219 \\
\hline $\bar{m}_{s}$ & Mass per unit length of slab & $\mathrm{kg} / \mathrm{m}$ & $1.2 \times 10^{3}$ \\
\hline$k_{r s y}$ & Lateral stiffness of fastener & $\mathrm{N} / \mathrm{m}$ & $3.0 \times 10^{7}$ \\
\hline$k_{r s z}$ & Vertical stiffness of fastener & $\mathrm{N} / \mathrm{m}$ & $5.0 \times 10^{7}$ \\
\hline $\bar{k}_{s b y}$ & Lateral stiffness of CAM per unit length & $\mathrm{N} / \mathrm{m}^{2}$ & $1.5 \times 10^{9}$ \\
\hline $\bar{k}_{s b z}$ & Vertical stiffness of CAM per unit length & $\mathrm{N} / \mathrm{m}^{2}$ & $1.5 \times 10^{9}$ \\
\hline \multicolumn{4}{|c|}{ 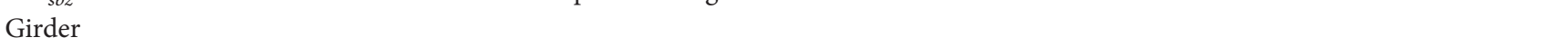 } \\
\hline$E_{b}$ & Young's modulus of girder & $\mathrm{N} / \mathrm{m}^{2}$ & $3.45 \times 10^{10}$ \\
\hline$I_{b y}$ & Flexural moment of inertia about $y$-axis of cross section of girder & $\mathrm{m}^{4}$ & 12.744 \\
\hline$I_{b z}$ & Flexural moment of inertia about $z$-axis of cross section of girder & $\mathrm{m}^{4}$ & 96.435 \\
\hline $\bar{m}_{b}$ & Mass per unit length of girder & $\mathrm{kg} / \mathrm{m}$ & $2.972 \times 10^{4}$ \\
\hline$\omega_{b 1}$ & Fundamental frequency of girder & $\mathrm{Hz}$ & 5.58 \\
\hline$\omega_{b 2}$ & Second natural frequency of girder & $\mathrm{Hz}$ & 13.26 \\
\hline \multicolumn{4}{|l|}{ Pier } \\
\hline$E_{p}$ & Young's modulus of pier & $\mathrm{N} / \mathrm{m}^{2}$ & $3.15 \times 10^{10}$ \\
\hline$I_{p x}$ & Flexural moment of inertia about $x$-axis of cross section of pier & $\mathrm{m}^{4}$ & 73.23 \\
\hline $\bar{m}_{p}$ & Mass per unit length of pier & $\mathrm{kg} / \mathrm{m}$ & $5.2 \times 10^{4}$ \\
\hline
\end{tabular}

$\zeta_{f z}=\zeta_{g z}, \omega_{f z}=0.1 \omega_{g z}-0.2 \omega_{g z}$, and $\mathbf{S}_{0 z}=0.218 \mathbf{S}_{0 y}$. The parameters for the earthquakes with different intensities and site types are listed in Table 2. It is assumed that the DBGA is $0.15 \mathrm{~g}$ and the bridge site is Type II. The spatial frequency of the track irregularity PSDs ranges from $0.004 \times 2 \pi$ to 1 $\times 2 \pi \mathrm{rad} / \mathrm{m}$, while the frequency of the seismic acceleration PSDs lies in the range of $0-40 \mathrm{~Hz}$. It is worth noting that the extreme state for the maximum responses of the bridge and each moving vehicle may not occur at the same time when the track irregularities and earthquakes are considered simultaneously, and therefore cannot be easily identified. This makes it rather difficult to determine how the earthquakes' starting times affect the results. Here, the earthquakes are assumed to start exactly at the instant when the train enters the bridge [17].

5.2. Analysis of the PSD Characteristic of the Random Vibration Responses of Bridge and Train. The random vibration responses of the bridge and train calculated by PEM with train speed of $300 \mathrm{~km} / \mathrm{h}$ are still used.

The PSDs of the vertical and lateral acceleration of the girder midpoint to the passage of the train shaken by track irregularities and earthquakes are plotted in Figure 4. It can be seen from Figure 4(a) that there exists only one dominant vibration frequency (DVF) of $5.34 \mathrm{~Hz}$, which is very close to the vertical fundamental frequency of the girder of $5.58 \mathrm{~Hz}$. Similarly, only one DVF of $3.07 \mathrm{~Hz}$ can be found from

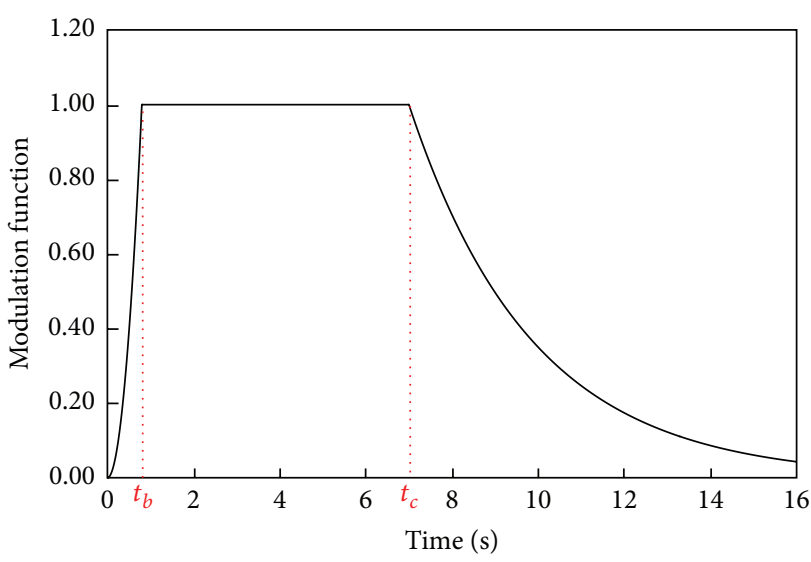

Figure 3: Plots of modulation function $\mathbf{g}(t)$.

Figure 4(b), which is very close to the lateral fundamental frequency of the total bridge (including the girder and pier) of $3.11 \mathrm{~Hz}$.

Figure 5 exhibit the PSDs of the vertical and lateral accelerations of the trailer carbody. As can be seen, the PSDs change violently with the vibration frequency, implying the great influence of track irregularities and earthquakes on the dynamic response of the carbody. Of interest is that there exist two DVFs with DVF1 $=0.83 \mathrm{~Hz}$ and DVF2 $=5.19 \mathrm{~Hz}$ 
TABle 2: Parameters of earthquake for different site conditions.

\begin{tabular}{|c|c|c|c|c|}
\hline \multirow{2}{*}{ Parameter } & \multirow{2}{*}{ Unit } & \multicolumn{3}{|c|}{ Site classification } \\
\hline & & Type I & Type II & Type III \\
\hline$\omega_{g y}$ & $\mathrm{rad} / \mathrm{s}$ & 25.13 & 17.95 & 13.96 \\
\hline$\zeta_{g y}$ & - & 0.64 & 0.72 & 0.80 \\
\hline$t_{b}$ & s & 0.5 & 0.8 & 1.2 \\
\hline$t_{c}$ & s & 5.5 & 7.0 & 9.0 \\
\hline$c$ & - & 0.45 & 0.35 & 0.25 \\
\hline Duration of acting time of earthquake & s & 6.69 & 8.41 & 10.92 \\
\hline $\mathbf{S}_{0 y}$ for $\mathrm{DBGA}=0.15 \mathrm{~g}^{* *}$ & $\mathrm{~cm}^{2} / \mathrm{s}^{3}$ & 275 & 364 & 440 \\
\hline $\mathrm{S}_{0 y}$ for $\mathrm{DBGA}=0.30 \mathrm{~g}^{* *}$ & $\mathrm{~cm}^{2} / \mathrm{s}^{3}$ & 1099 & 1456 & 1759 \\
\hline $\mathbf{S}_{0 y}$ for DBGA $=0.40 \mathrm{~g}^{* *}$ & $\mathrm{~cm}^{2} / \mathrm{s}^{3}$ & 1780 & 2359 & 2849 \\
\hline
\end{tabular}

${ }^{* *}$ DBGA is the design basic acceleration of ground motion.

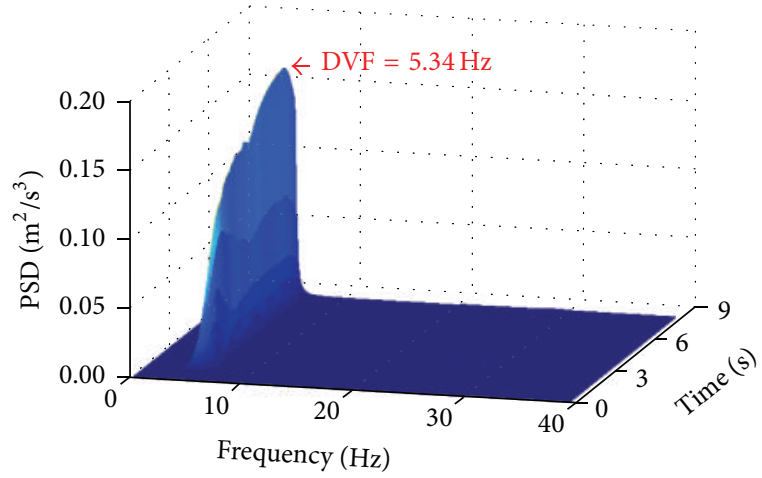

(a)

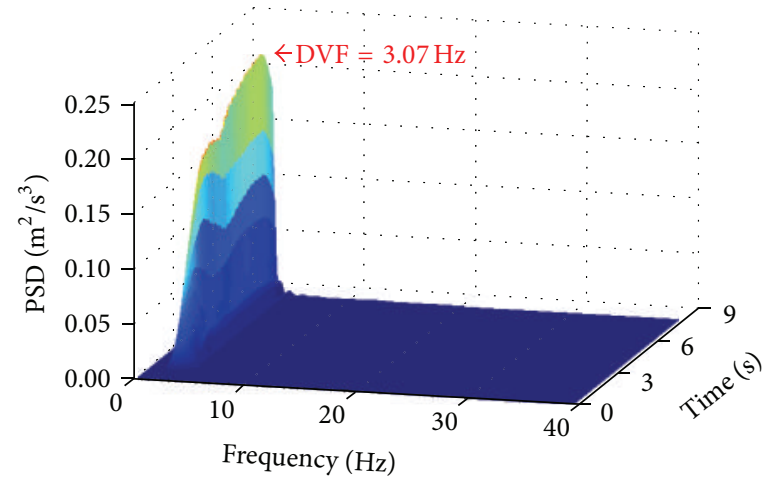

(b)

FIGURE 4: PSD of acceleration of bridge girder midpoint. (a) Vertical. (b) Lateral.

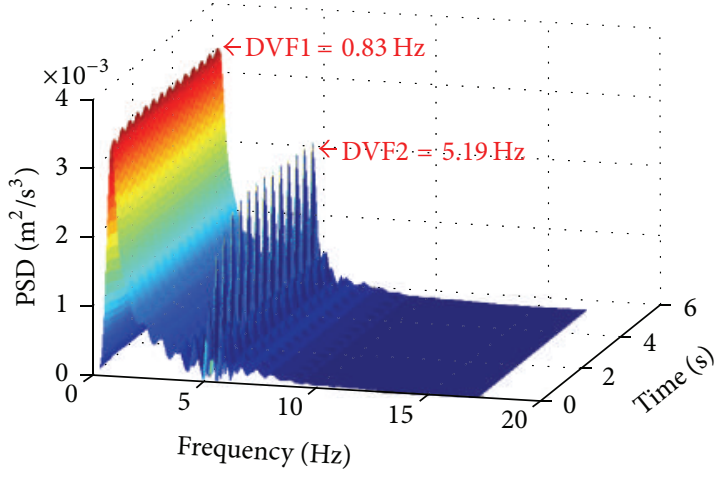

(a)

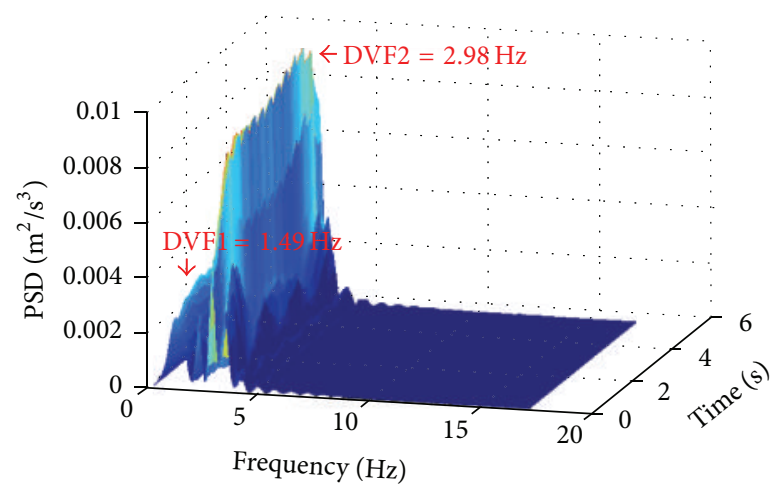

(b)

FIGURE 5: PSD of acceleration of trailer carbody. (a) Vertical. (b) Lateral.

in Figure 5(a), which are approximately equal to the vertical fundamental frequency of the trailer carbody and that of the girder, respectively. Similarly, two DVFs with DVF1 $=1.49 \mathrm{~Hz}$ and DVF2 $=2.98 \mathrm{~Hz}$ can be seen easily from Figure 5(b), which are also close to the lateral fundamental frequency of the trailer carbody and of the total bridge, respectively. Obviously, the vertical DVF1 and the lateral DVF1 are mainly excited by track irregularities, while the vertical DVF2 and the lateral DVF2 are mainly induced by the bridge vibration under the shake of earthquakes. On the other hand, the vertical and lateral PSDs excited by track irregularities vary only slightly with the time, which indicates that the influence of the bridge vibration on the carbody vibration is insignificant if there is no earthquake, because of the comparatively high mass and flexural rigidity of the bridge. However, the vertical PSD induced by earthquakes varies periodically with 


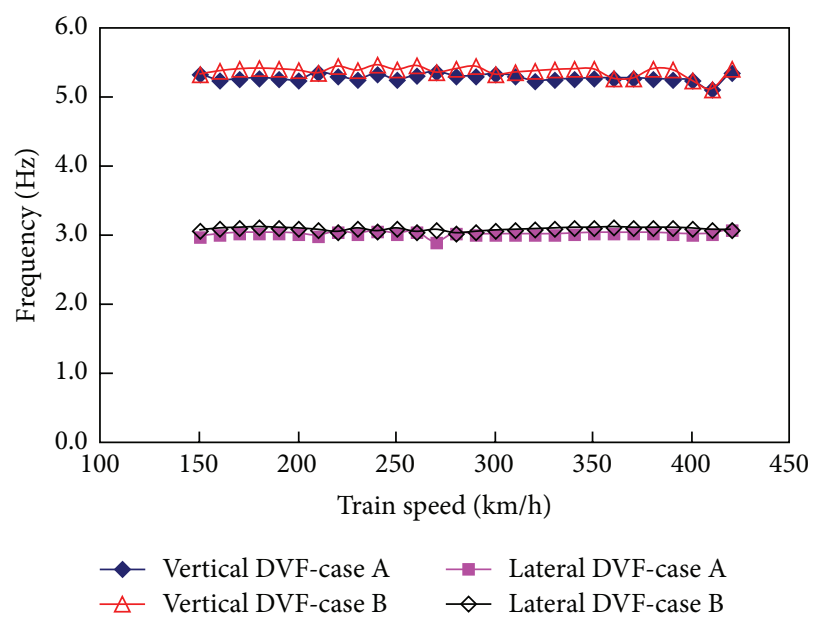

(a)

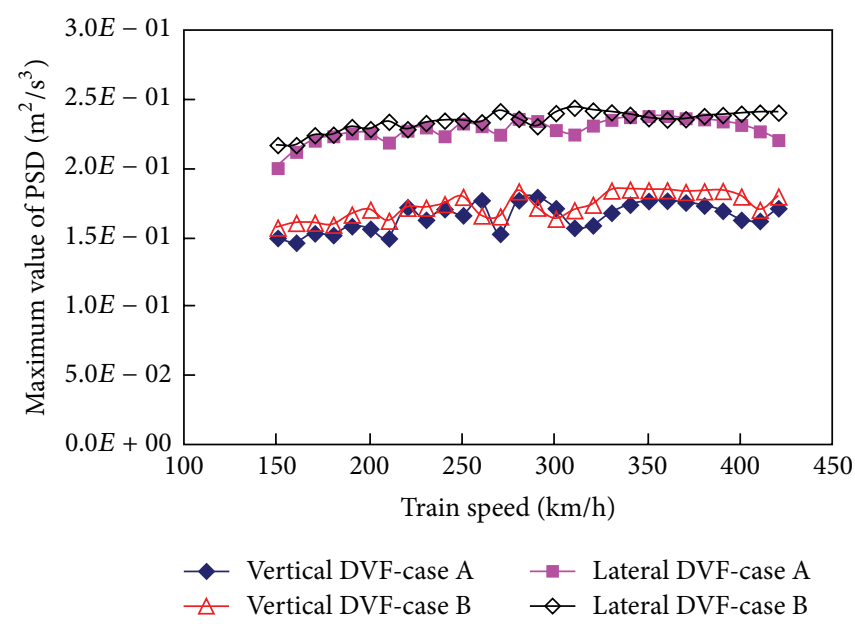

(b)

FIGURE 6: Girder midpoint acceleration with respect to train speed. (a) DVF. (b) Maximum value of PSD.

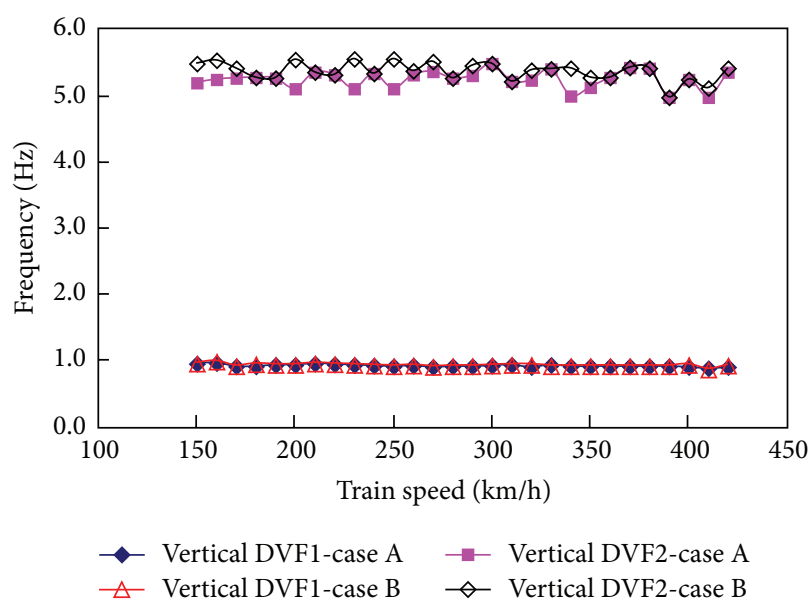

(a)

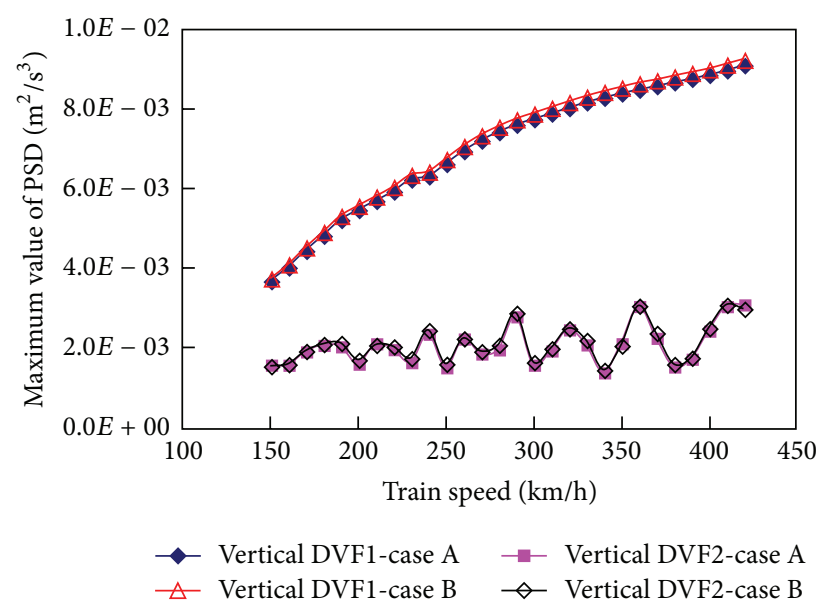

(b)

FIGURE 7: Motor carbody vertical acceleration with respect to train speed. (a) DVF. (b) Maximum value of PSD.

the vehicle passing through different girder (Figure 5(a)), while similar trend cannot be found for the lateral PSD induced by earthquakes (Figure 5(b)).

A similar phenomenon for the vertical and lateral accelerations of the motor carbody can be also observed. Herein it is not discussed in detail to save the length of the paper.

5.3. Influence of Train Speed on the Random Vibration Characteristic of Bridge and Train. In reality, the train may move over the bridge at various speeds during earthquakes. There exists a need to investigate the random vibration characteristic of the train moving over the bridge under various train speeds, as they may be different. The train is assumed to move over the bridge with a constant speed varying from 150 to $420 \mathrm{~km} / \mathrm{h}$ at $10 \mathrm{~km} / \mathrm{h}$ intervals. The other parameters are the same as listed in Section 5.1. Furthermore, two calculation cases (Table 3 ) are computed for comparison. In case B, the relative displacements between wheel, track, and girder are
TABLE 3: Calculation cases for studying the influence of train speed.

\begin{tabular}{lcc}
\hline Calculation case & A & B \\
\hline Calculation model & TSTBI model & $\begin{array}{c}\text { Train-bridge } \\
\text { interaction model [6] }\end{array}$ \\
\hline Excitations & $\begin{array}{c}\text { Track irregularity } \\
+ \text { earthquake }\end{array}$ & $\begin{array}{c}\text { Track irregularity } \\
+ \text { earthquake }\end{array}$ \\
\hline
\end{tabular}

neglected [6]. The maximum values of the PSD and the DVF of the bridge and train with respect to train speed are shown in Figures 6-10 and Tables 4 and 5.

Some conclusions can be drawn from Figure 6 and Table 4: (1) both the maximum values of the PSD and the DVF of the girder vary slightly as the train speed increases in both cases $\mathrm{A}$ and $\mathrm{B}$, which indicates that the vertical and lateral vibrations of the girder are induced basically by earthquakes and not by the train and the track irregularities. 
TABLE 4: Influence of train speed on PSDs of bridge midpoint acceleration.

\begin{tabular}{|c|c|c|c|c|c|c|c|c|}
\hline \multirow{3}{*}{$\begin{array}{l}\text { Train speed } \\
(\mathrm{km} / \mathrm{h})\end{array}$} & \multicolumn{4}{|c|}{ Vertical } & \multicolumn{4}{|c|}{ Lateral } \\
\hline & \multicolumn{2}{|c|}{$\mathrm{DVF}(\mathrm{Hz})$} & \multicolumn{2}{|c|}{ Peak value $\left(\mathrm{m}^{2} / \mathrm{s}^{3}\right)$} & \multicolumn{2}{|c|}{$\operatorname{DVF}(\mathrm{Hz})$} & \multicolumn{2}{|c|}{ Peak value $\left(\mathrm{m}^{2} / \mathrm{s}^{3}\right)$} \\
\hline & Case A & Case B & Case A & Case B & Case A & Case B & Case A & Case B \\
\hline 150 & 5.34 & 5.34 & $1.50 E-01$ & $1.58 E-01$ & 2.98 & 3.07 & $2.01 E-01$ & $2.17 E-01$ \\
\hline 170 & 5.27 & 5.42 & $1.53 E-01$ & $1.61 E-01$ & 3.03 & 3.11 & $2.20 E-01$ & $2.24 E-01$ \\
\hline 190 & 5.27 & 5.42 & $1.59 E-01$ & $1.67 E-01$ & 3.03 & 3.11 & $2.26 E-01$ & $2.30 E-01$ \\
\hline 210 & 5.36 & 5.36 & $1.50 E-01$ & $1.63 E-01$ & 2.99 & 3.08 & $2.19 E-01$ & $2.34 E-01$ \\
\hline 230 & 5.26 & 5.40 & $1.63 E-01$ & $1.72 E-01$ & 3.02 & 3.10 & $2.30 E-01$ & $2.33 E-01$ \\
\hline 250 & 5.26 & 5.41 & $1.66 E-01$ & $1.80 E-01$ & 3.02 & 3.10 & $2.33 E-01$ & $2.35 E-01$ \\
\hline 270 & 5.37 & 5.37 & $1.53 E-01$ & $1.66 E-01$ & 2.90 & 3.08 & $2.25 E-01$ & $2.42 E-01$ \\
\hline 290 & 5.31 & 5.46 & $1.80 E-01$ & $1.72 E-01$ & 3.01 & 3.05 & $2.35 E-01$ & $2.31 E-01$ \\
\hline 310 & 5.31 & 5.37 & $1.57 E-01$ & $1.70 E-01$ & 3.01 & 3.08 & $2.25 E-01$ & $2.44 E-01$ \\
\hline 330 & 5.26 & 5.41 & $1.68 E-01$ & $1.84 E-01$ & 3.01 & 3.10 & $2.36 E-01$ & $2.41 E-01$ \\
\hline 350 & 5.28 & 5.42 & $1.77 E-01$ & $1.85 E-01$ & 3.03 & 3.11 & $2.38 E-01$ & $2.37 E-01$ \\
\hline 370 & 5.28 & 5.28 & $1.76 E-01$ & $1.83 E-01$ & 3.03 & 3.11 & $2.37 E-01$ & $2.36 E-01$ \\
\hline 390 & 5.26 & 5.41 & $1.70 E-01$ & $1.84 E-01$ & 3.02 & 3.11 & $2.34 E-01$ & $2.39 E-01$ \\
\hline 410 & 5.12 & 5.12 & $1.62 E-01$ & $1.70 E-01$ & 3.02 & 3.08 & $2.27 E-01$ & $2.41 E-01$ \\
\hline
\end{tabular}

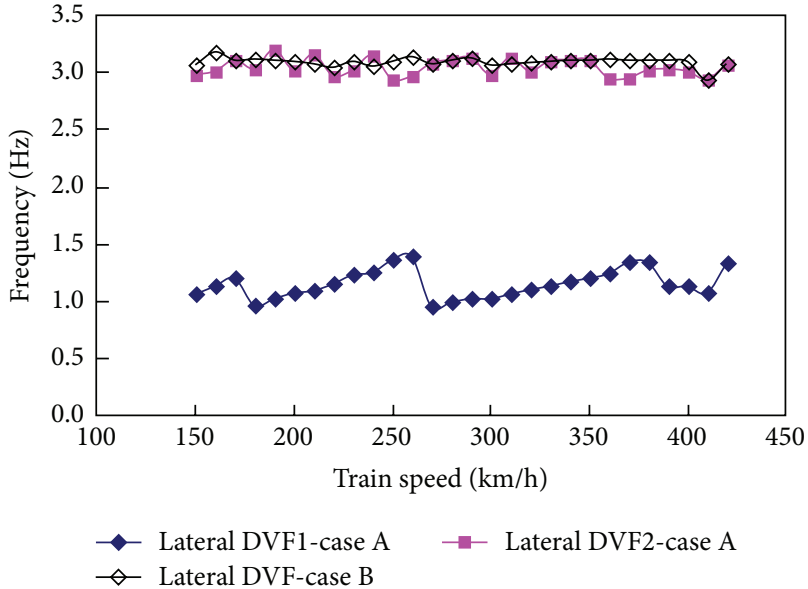

(a)

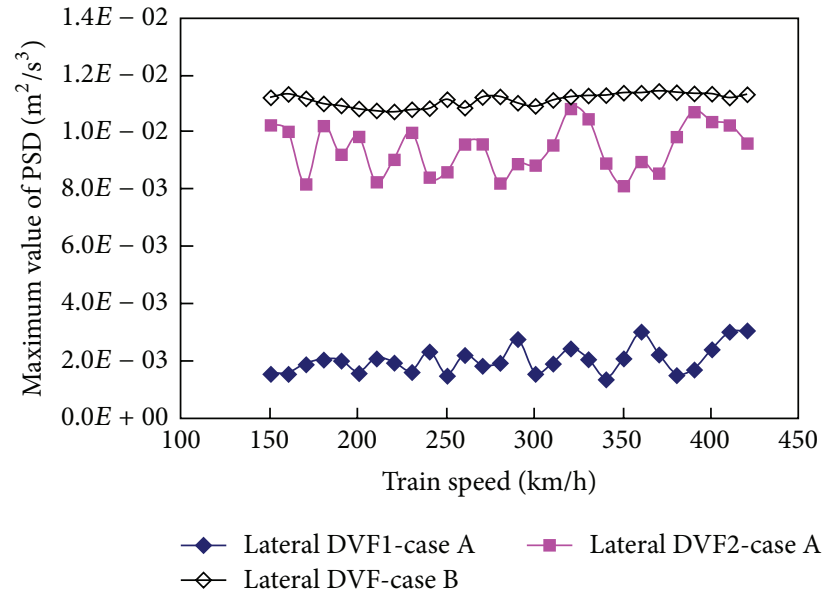

(b)

FIGURE 8: Motor carbody lateral acceleration with respect to train speed. (a) DVF. (b) Maximum value of PSD.

(2) The maximum values of the PSD in case B tend to be larger than those in case $A$ because the effects of the constraint of the track are not taken into account in case B, but the degree of increase is below $10 \%$.

From Figures 7-10 and Table 5, the following observations can be made easily: (1) the DVF1 and DVF2 for both the vertical and lateral accelerations of the carbody change slightly around the corresponding fundamental frequencies of the train and bridge with the rising of train speed in both cases A and B. (2) For the carbody vertical acceleration, the maximum values of the PSD of DVF1 increase as the train speed increases in both cases A and B. (3) The maximum values of the PSD of DVF1 for the carbody lateral acceleration do not show a trend of monotonic increase for higher train speeds in case A. This trend can be also found from the maximum values of the PSD of DVF2 for both the vertical and lateral accelerations of the carbody in case A. (4) For the carbody vertical acceleration, the maximum values of the PSD of DVF1 are generally bigger than those of DVF2 in both cases A and B, implying that the influence of the track irregularities in the vertical direction is more obvious than that of earthquakes. However the opposite is the case for the carbody lateral acceleration. (5) Unlike in case A, there exists only one DVF for the lateral accelerations of the carbody in case $\mathrm{B}$, which generally coincides with the lateral fundamental frequency of the bridge. Meanwhile, the peak values of the PSD of the carbody acceleration in case B are generally larger than those in case A, but the difference between the lateral accelerations of the carbody is much larger than that for the vertical accelerations. For the motor 


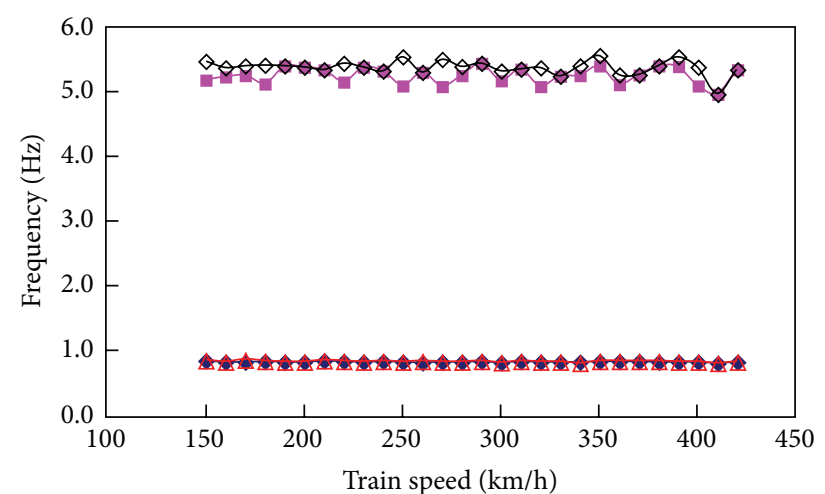

$\multimap$ Vertical DVF1-case A $\rightarrow$ Vertical DVF2-case A
$\triangle$ Vertical DVF1-case B $\rightarrow$ Vertical DVF2-case B

(a)

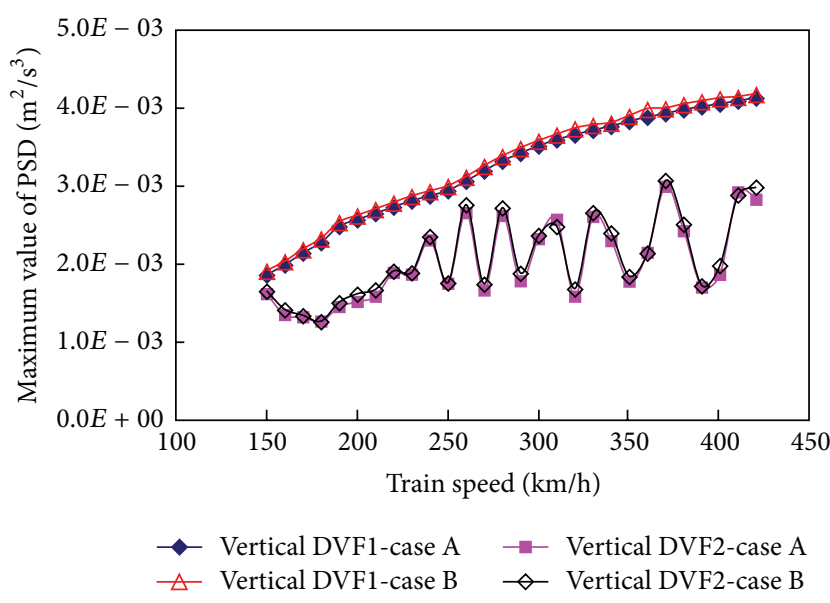

(b)

FIGURE 9: Trailer carbody vertical acceleration with respect to train speed. (a) DVF. (b) Maximum value of PSD.

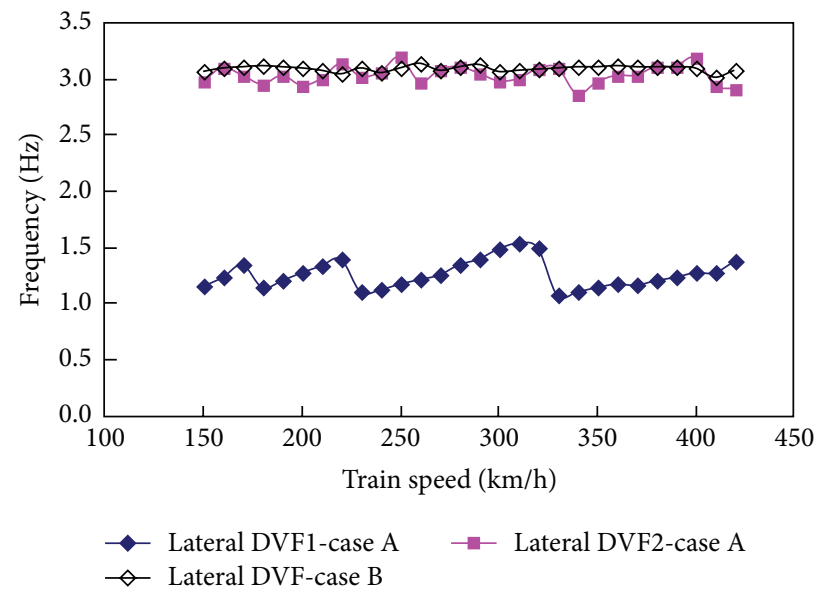

(a)

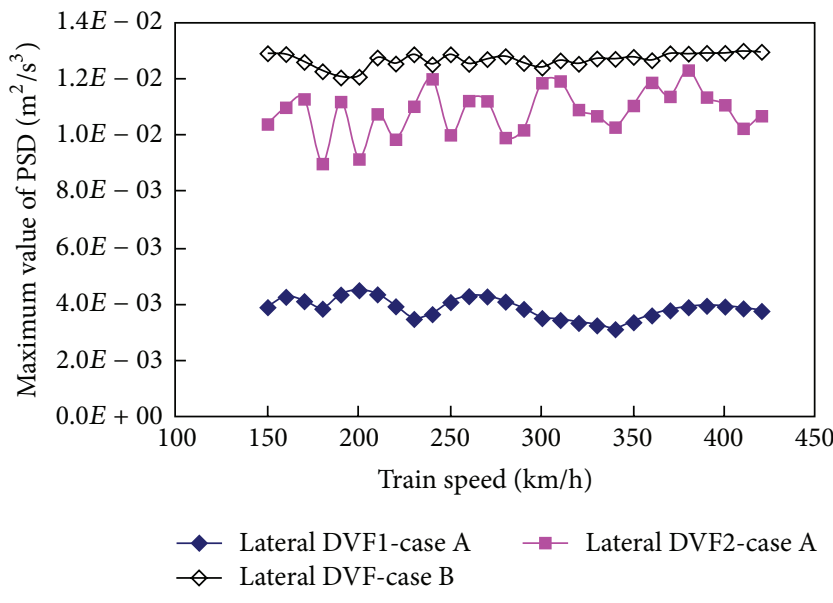

(b)

FIGURE 10: Trailer carbody lateral acceleration with respect to train speed. (a) DVF. (b) Maximum value of PSD.

car, the lateral and vertical accelerations of case B are larger than those of case A by up to $36 \%$ and $6 \%$, respectively. One reason behind these is that the influence of earthquakes on the carbody lateral accelerations is greater than that of track irregularities. Another reason is that the inherent relative displacements between the wheel and track in the lateral direction are probably much larger than that in the vertical direction in case A.

5.4. Analysis of Dynamic Reliability of Train Operation Stability. As was mentioned in [7], the train operation stability over bridges under the action of earthquakes is an important subject for the railway engineers because the moving trains may not be safe enough due to the excessive vibrations of bridges. The dynamic reliability of train operation stability is investigated in this paper according to (35), in which " $b$ " is equal to the allowable safety limit of each evaluation index. The standards used for evaluation of train operation stability are listed in Table 6 [38].
Three numerical examples are applied to analyze the influence of the train speed, the earthquake intensity, and the pier height on the dynamic reliability of train operation stability, respectively. To investigate the influence of the train speed on the dynamic reliability of train operation stability over bridge, the train is also assumed to move over the bridge with a constant speed varying from 150 to $420 \mathrm{~km} / \mathrm{h}$ at $10 \mathrm{~km} / \mathrm{h}$ intervals, with the same track irregularities and earthquakes as listed in Section 5.1. The estimated dynamic reliability of train operation stability with respect to train speed is plotted in Figures 11-14. To investigate the influence of the earthquake intensity on the dynamic reliability of train operation stability over bridge, seven lateral seismic accelerations varying from $0.0 \mathrm{~g}$ to $0.3 \mathrm{~g}$ are used as the input excitations, respectively. For each case, the train is assumed to pass through the bridge at a speed of $300 \mathrm{~km} / \mathrm{h}$. The other parameters are as for Section 5.1. The estimated dynamic reliability of train operation stability with respect to seismic acceleration is shown in Figures 15-18. To investigate the influence of 
TABLE 5: Influence of train speed on PSDs of motor carbody acceleration.

\begin{tabular}{|c|c|c|c|c|c|c|c|c|}
\hline \multirow{2}{*}{$\begin{array}{l}\text { Train speed } \\
(\mathrm{km} / \mathrm{h})\end{array}$} & \multicolumn{4}{|c|}{ Vertical } & \multicolumn{4}{|c|}{ Lateral } \\
\hline & $\begin{array}{l}\text { DVF1 } \\
(\mathrm{Hz})\end{array}$ & $\begin{array}{c}\text { Peak value } \\
\left(\mathrm{m}^{2} / \mathrm{s}^{3}\right)\end{array}$ & $\begin{array}{c}\text { DVF2 } \\
(\mathrm{Hz})\end{array}$ & $\begin{array}{c}\text { Peak value } \\
\left(\mathrm{m}^{2} / \mathrm{s}^{3}\right)\end{array}$ & $\begin{array}{c}\text { DVF1 } \\
(\mathrm{Hz})\end{array}$ & $\begin{array}{c}\text { Peak value } \\
\left(\mathrm{m}^{2} / \mathrm{s}^{3}\right)\end{array}$ & $\begin{array}{c}\text { DVF2 } \\
(\mathrm{Hz})\end{array}$ & $\begin{array}{c}\text { Peak value } \\
\left(\mathrm{m}^{2} / \mathrm{s}^{3}\right)\end{array}$ \\
\hline \multicolumn{9}{|l|}{150} \\
\hline Case A & 0.96 & $3.69 E-03$ & 5.20 & $1.59 E-03$ & 1.07 & $1.59 E-03$ & 2.98 & $1.03 E-02$ \\
\hline Case B & 0.96 & $3.75 E-03$ & 5.49 & $1.55 E-03$ & - & - & 3.07 & $1.12 E-02$ \\
\hline \multicolumn{9}{|l|}{170} \\
\hline Case A & 0.92 & $4.44 E-03$ & 5.27 & $1.92 E-03$ & 1.21 & $1.92 E-03$ & 3.11 & $8.21 E-03$ \\
\hline Case B & 0.92 & $4.53 E-03$ & 5.42 & $1.93 E-03$ & - & - & 3.11 & $1.12 E-02$ \\
\hline \multicolumn{9}{|l|}{190} \\
\hline Case A & 0.94 & $5.22 E-03$ & 5.27 & $2.05 E-03$ & 1.03 & $2.05 E-03$ & 3.20 & $9.24 E-03$ \\
\hline Case B & 0.94 & $5.32 E-03$ & 5.27 & $2.13 E-03$ & - & - & 3.11 & $1.09 E-02$ \\
\hline \multicolumn{9}{|l|}{210} \\
\hline Case A & 0.96 & $5.70 E-03$ & 5.36 & $2.12 E-03$ & 1.10 & $2.12 E-03$ & 3.16 & $8.28 E-03$ \\
\hline Case B & 0.96 & $5.81 E-03$ & 5.36 & $2.08 E-03$ & - & - & 3.08 & $1.08 E-02$ \\
\hline \multicolumn{9}{|l|}{230} \\
\hline Case A & 0.94 & $6.23 E-03$ & 5.11 & $1.65 E-03$ & 1.24 & $1.65 E-03$ & 3.02 & $1.00 E-02$ \\
\hline Case B & 0.94 & $6.35 E-03$ & 5.56 & $1.75 E-03$ & - & - & 3.10 & $1.08 E-02$ \\
\hline \multicolumn{9}{|l|}{250} \\
\hline Case A & 0.92 & $6.62 E-03$ & 5.11 & $1.52 E-03$ & 1.37 & $1.52 E-03$ & 2.94 & $8.63 E-03$ \\
\hline Case B & 0.92 & $6.75 E-03$ & 5.56 & $1.61 E-03$ & - & - & 3.10 & $1.12 E-02$ \\
\hline \multicolumn{9}{|l|}{270} \\
\hline Case A & 0.91 & $7.21 E-03$ & 5.37 & $1.87 E-03$ & 0.96 & $1.87 E-03$ & 3.08 & $9.61 E-03$ \\
\hline Case B & 0.91 & $7.34 E-03$ & 5.52 & $1.93 E-03$ & - & - & 3.08 & $1.12 E-02$ \\
\hline \multicolumn{9}{|l|}{290} \\
\hline Case A & 0.92 & $7.61 E-03$ & 5.31 & $2.80 E-03$ & 1.03 & $2.80 E-03$ & 3.13 & $8.92 E-03$ \\
\hline Case B & 0.92 & $7.74 E-03$ & 5.46 & $2.89 E-03$ & - & - & 3.13 & $1.10 E-02$ \\
\hline \multicolumn{9}{|l|}{310} \\
\hline Case A & 0.94 & $7.89 E-03$ & 5.22 & $1.95 E-03$ & 1.07 & $1.95 E-03$ & 3.13 & $9.57 E-03$ \\
\hline Case B & 0.94 & $8.03 E-03$ & 5.22 & $2.00 E-03$ & - & - & 3.08 & $1.11 E-02$ \\
\hline \multicolumn{9}{|l|}{330} \\
\hline Case A & 0.94 & $8.17 E-03$ & 5.41 & $2.10 E-03$ & 1.14 & $2.10 E-03$ & 3.10 & $1.05 E-02$ \\
\hline Case B & 0.92 & $8.31 E-03$ & 5.41 & $2.21 E-03$ & - & - & 3.10 & $1.13 E-02$ \\
\hline \multicolumn{9}{|l|}{350} \\
\hline Case A & 0.92 & $8.39 E-03$ & 5.13 & $2.13 E-03$ & 1.21 & $2.13 E-03$ & 3.11 & $8.15 E-03$ \\
\hline Case B & 0.92 & $8.54 E-03$ & 5.28 & $2.07 E-03$ & - & - & 3.11 & $1.14 E-02$ \\
\hline \multicolumn{9}{|l|}{370} \\
\hline Case A & 0.92 & $8.58 E-03$ & 5.43 & $2.26 E-03$ & 1.35 & $2.26 E-03$ & 2.95 & $8.59 E-03$ \\
\hline Case B & 0.92 & $8.72 E-03$ & 5.43 & $2.38 E-03$ & - & - & 3.11 & $1.15 E-02$ \\
\hline \multicolumn{9}{|l|}{390} \\
\hline Case A & 0.92 & $8.76 E-03$ & 4.98 & $1.74 E-03$ & 1.14 & $1.74 E-03$ & 3.03 & $1.07 E-02$ \\
\hline Case B & 0.92 & $8.91 E-03$ & 4.98 & $1.76 E-03$ & - & - & 3.11 & $1.14 E-02$ \\
\hline \multicolumn{9}{|l|}{410} \\
\hline Case A & 0.89 & $8.97 E-03$ & 4.98 & $3.06 E-03$ & 1.08 & $3.06 E-03$ & 2.94 & $1.03 E-02$ \\
\hline Case B & 0.87 & $9.12 E-03$ & 5.12 & $3.09 E-03$ & - & - & 2.94 & $1.12 E-02$ \\
\hline
\end{tabular}


TABLE 6: Evaluation standards for train operation stability.

\begin{tabular}{lcc}
\hline Evaluation indices & Unit & Allowable limit \\
\hline $\begin{array}{l}\text { Carbody vertical } \\
\text { acceleration }\end{array}$ & $\mathrm{m} / \mathrm{s}^{2}$ & 1.30 \\
\hline $\begin{array}{l}\text { Carbody lateral } \\
\text { acceleration }\end{array}$ & $\mathrm{m} / \mathrm{s}^{2}$ & 1.00 \\
\hline $\begin{array}{l}\text { Lateral wheelset force } \\
\text { for motor car }\end{array}$ & $\mathrm{kN}$ & $53.83\left(=0.85 \times\left(10+P_{w} / 3\right)\right)^{* * *}$ \\
\hline $\begin{array}{l}\text { Lateral wheelset force } \\
\text { for trailer car }\end{array}$ & $\mathrm{kN}$ & $49.87\left(=0.85 \times\left(10+P_{w} / 3\right)\right)^{* * *}$ \\
\hline $\begin{array}{l}\text { Wheel load } \\
\text { decrement ratio }\end{array}$ & - & 0.65 \\
\hline
\end{tabular}

${ }^{* * *} P_{w}$ denotes the static wheelset load, which is equal to $160.0 \mathrm{kN}$ and $146.0 \mathrm{kN}$ for motor car and trailer car in this paper, respectively.

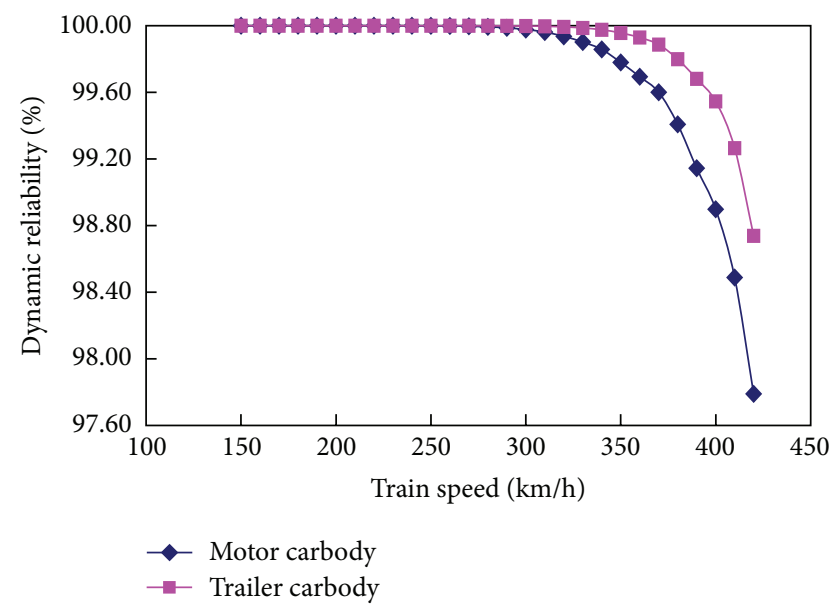

FIGURE 11: Dynamic reliability for carbody vertical acceleration with respect to train speed.

the pier height on the dynamic reliability of train operation stability over bridge, the analysis is performed by applying the height of pier from $10 \mathrm{~m}$ to $40 \mathrm{~m}$ at $2 \mathrm{~m}$ intervals and train running at a speed of $300 \mathrm{~km} / \mathrm{h}$, with other parameters same as those listed in Section 5.1. Figures 19-22 show the estimated dynamic reliability of train operation stability with respect to pier height.

The following observations can be drawn from Figures 1114: (1) In general, the dynamic reliability decrease with the increase of train speed. (2) The dynamic reliability for the vertical acceleration of both the motor carbody and the trailer carbody as well as for the wheel load decrement ratio of the motor car change relatively slowly. (3) The dynamic reliability for the carbody vertical acceleration and the lateral wheelset force reduces drastically as the train speed exceeds $300 \mathrm{~km} / \mathrm{h}$. (4) The dynamic reliability for the motor carbody acceleration decreases faster than that of the trailer carbody as the train speed increases. (5) The dynamic reliability for the wheel load decrement of the trailer car drops rapidly as the train speed exceeds $350 \mathrm{~km} / \mathrm{h}$.

The following are conclusions made from Figures 15-18: (1) as is expected, the dynamic reliability decreases generally with the increase of earthquake intensity. (2) The dynamic

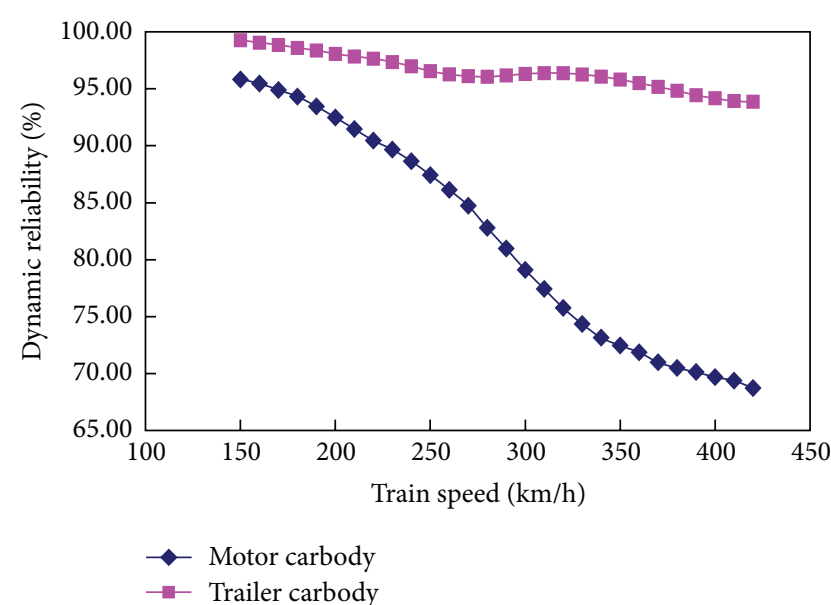

FIGURE 12: Dynamic reliability for carbody lateral acceleration with respect to train speed.

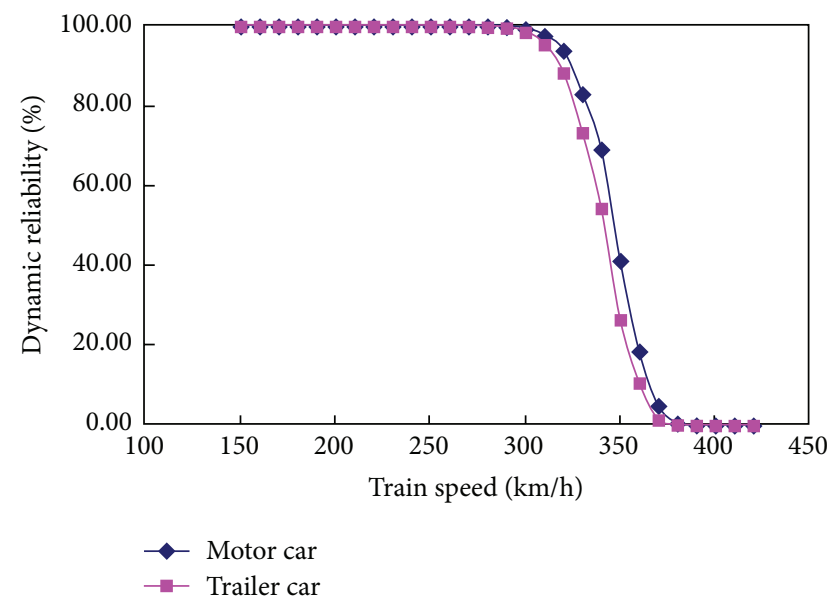

FIGURE 13: Dynamic reliability for lateral wheelset force with respect to train speed.

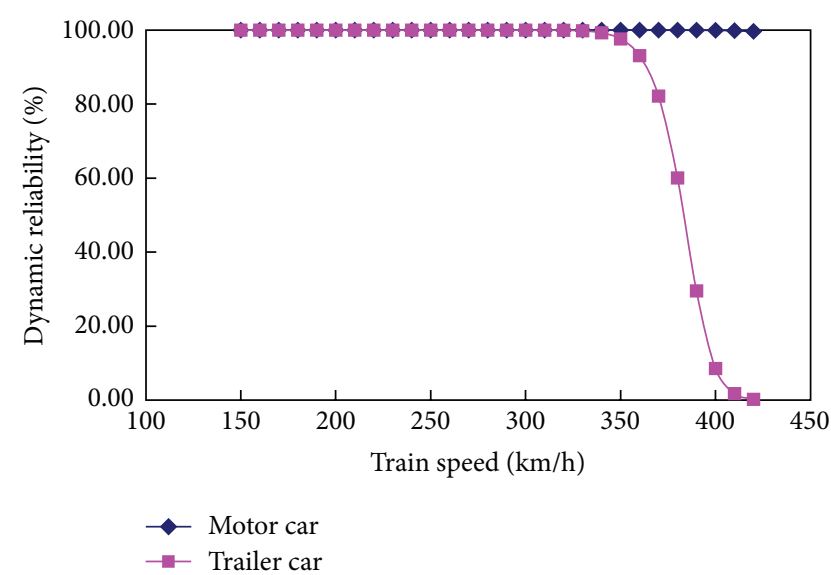

FIGURE 14: Dynamic reliability for wheel load decrement ratio with respect to train speed. 


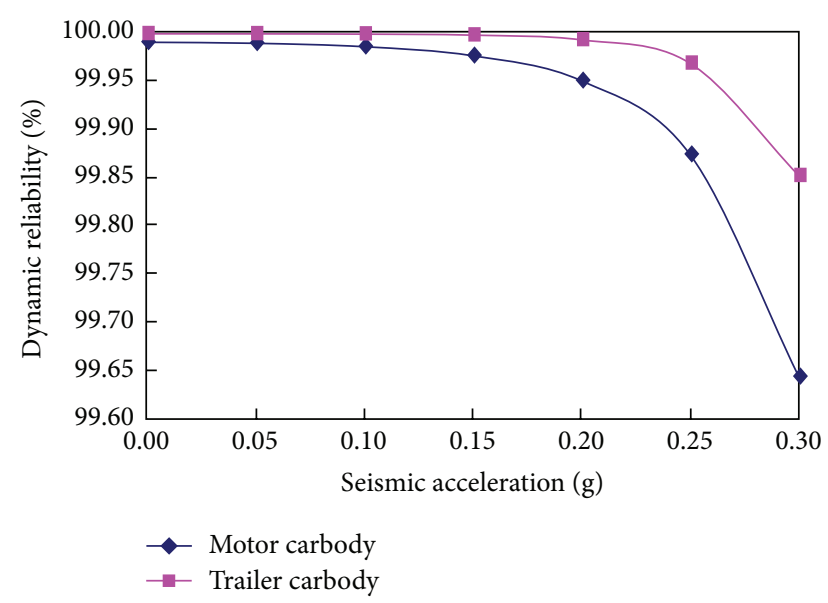

FIGURE 15: Dynamic reliability for carbody vertical acceleration with respect to seismic acceleration.

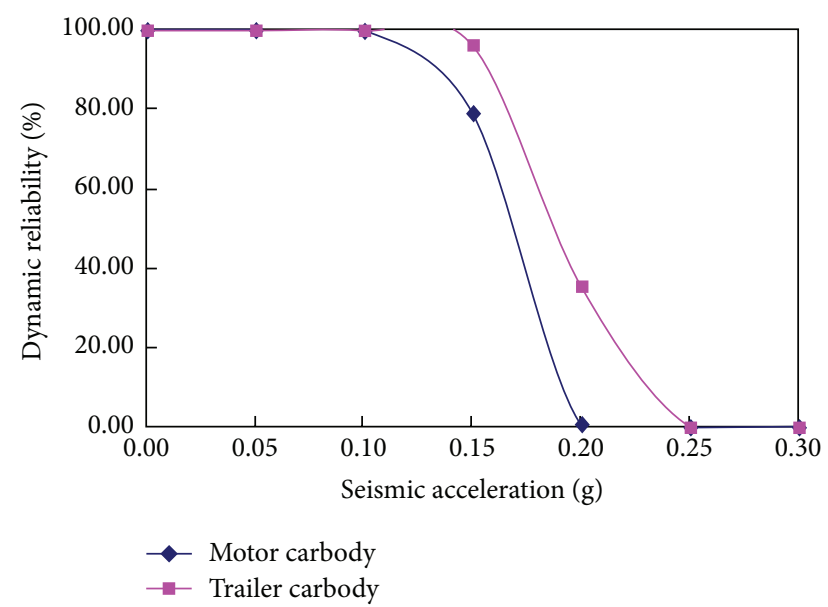

FIGURE 16: Dynamic reliability for carbody lateral acceleration with respect to seismic acceleration.

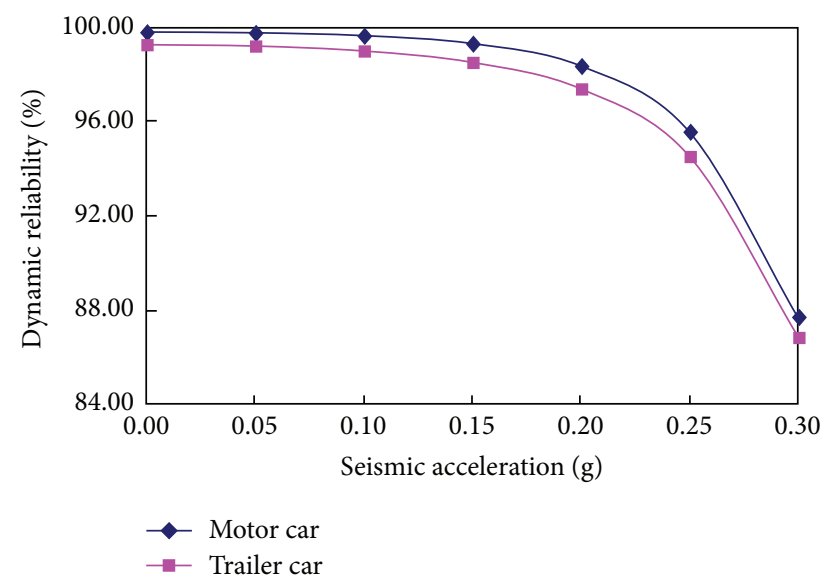

FIGURE 17: Dynamic reliability for lateral wheelset force with respect to seismic acceleration.

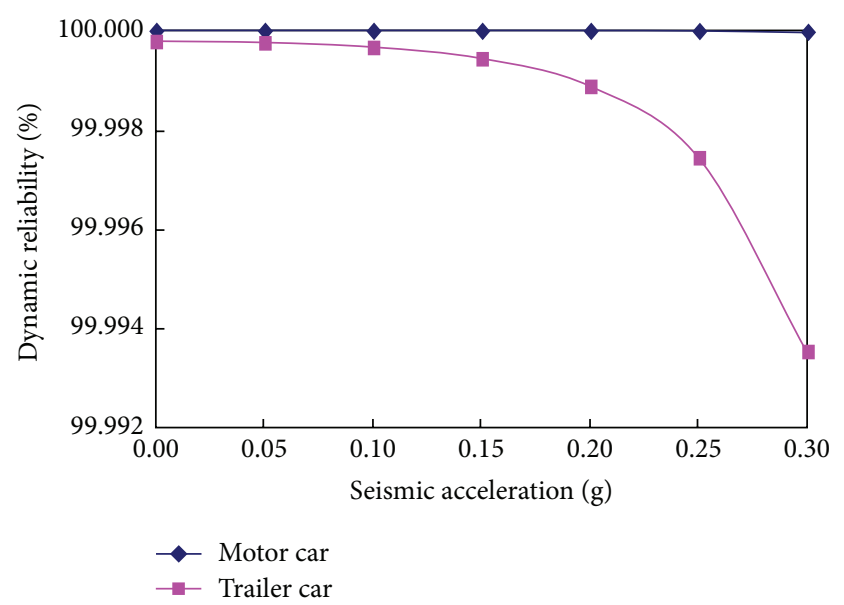

FIGURE 18: Dynamic reliability for wheel load decrement ratio with respect to seismic acceleration.

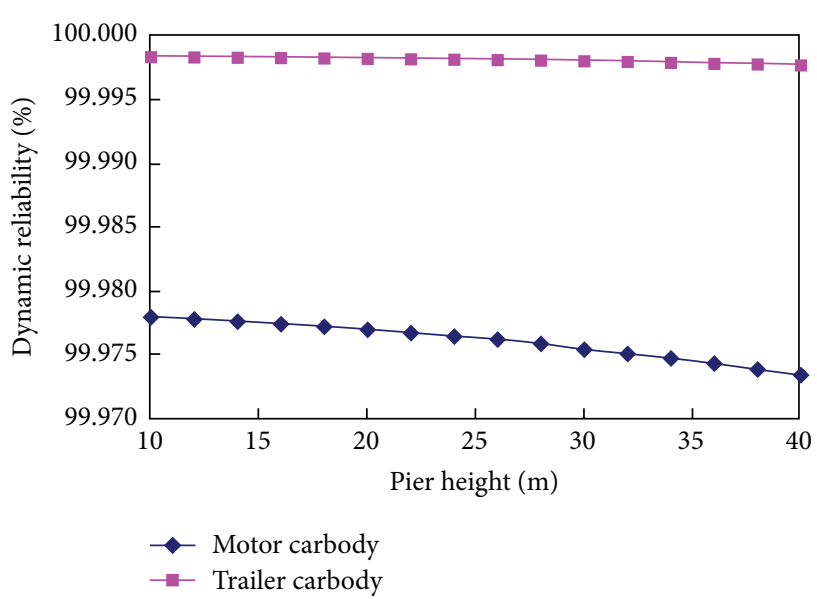

FIGURE 19: Dynamic reliability for carbody vertical acceleration with respect to pier height.

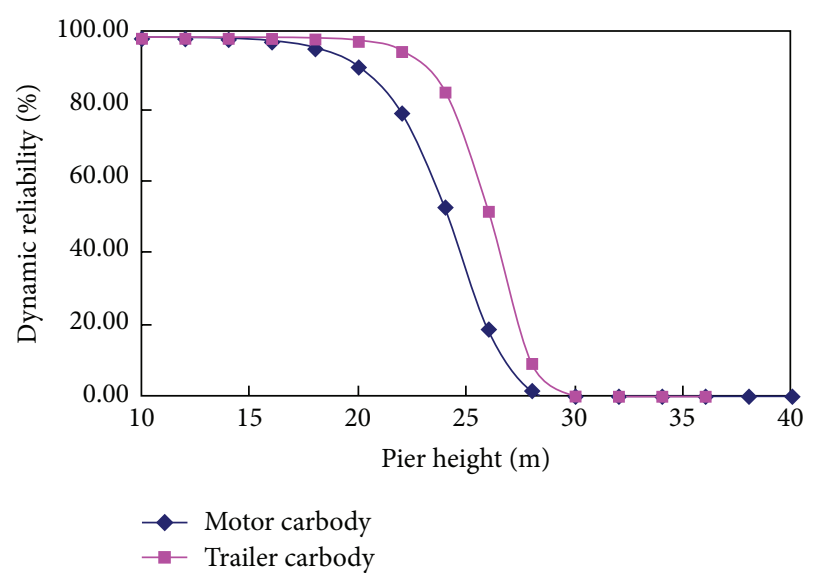

FIGURE 20: Dynamic reliability for carbody lateral acceleration with respect to pier height. 


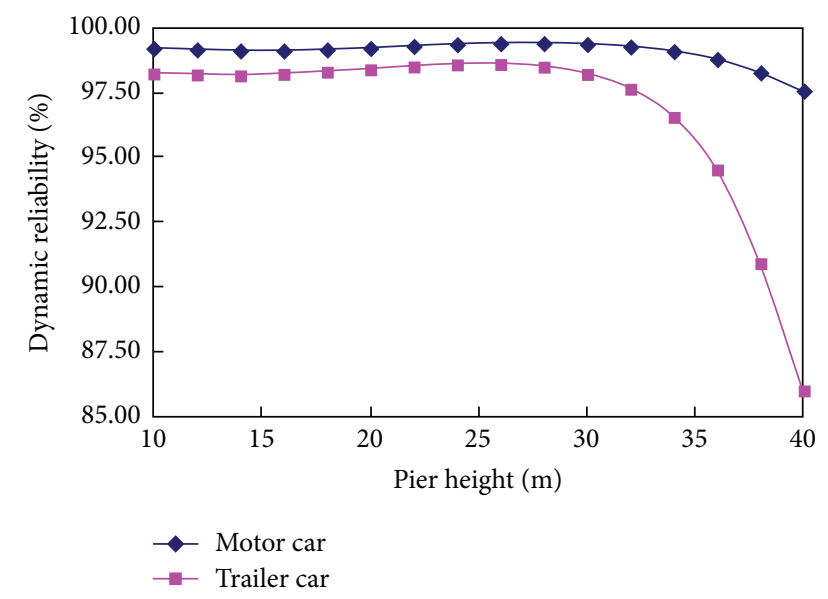

FIGURE 21: Dynamic reliability for lateral wheelset force with respect to pier height.

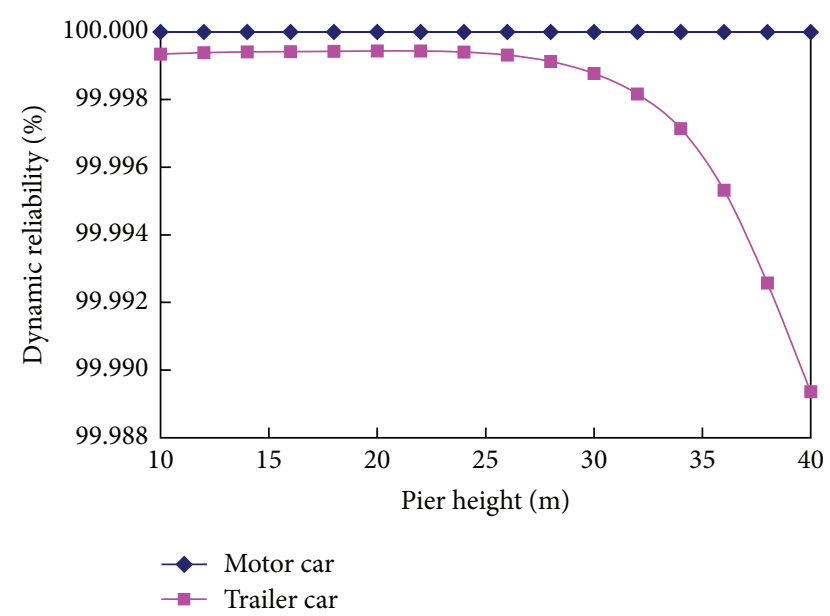

Figure 22: Dynamic reliability for wheel load decrement ratio with respect to pier height.

reliability related to the carbody lateral acceleration drops more rapidly than that related to the carbody vertical acceleration. (3) The dynamic reliability for the lateral acceleration of the motor carbody and the trailer carbody begins to decrease rapidly as the seismic acceleration reaches $0.10 \mathrm{~g}$ and $0.15 \mathrm{~g}$, respectively. (4) The dynamic reliability for the lateral wheelset force of both the motor car and the trailer car begins to drop quickly as the seismic acceleration reaches $0.20 \mathrm{~g}$.

Some conclusions can be drawn from Figures 19-22: (1) in general, the dynamic reliability also decreases with the increase of pier height. (2) Compared with the very slight change of the dynamic reliability related to the carbody vertical acceleration, the dynamic reliability related to the carbody lateral acceleration drops remarkably with the increasing of pier height. (3) The dynamic reliability for the lateral acceleration of the motor carbody and the trailer carbody begins to drop rapidly as the pier height reaches $16 \mathrm{~m}$ and $22 \mathrm{~m}$, respectively. (4) The dynamic reliability for the lateral wheelset force of the trailer car begins to decrease quickly as the pier height reaches $30 \mathrm{~m}$.

\section{Summary}

In this study, the three-dimensional vibration model of the TSTBI system is firstly established by FEM, the equations of motion for the entire system are then derived by means of energy principle. The excitations caused by random track irregularities and seismic accelerations are transformed into a series of deterministic pseudoharmonic excitation vectors via PEM. Taking a high-speed train traveling over a fifteenspan simply supported girder bridge as an example, the random vibration characteristic of the coupled system and the dynamic reliability of train operation stability are investigated. From the numerical results obtained in this work, the following conclusions can be drawn:

(1) The time-dependent PSDs of the random vibration responses of the TSTBI system simultaneously excited by track irregularities and earthquakes can be obtained easily by PEM, which may be useful for unveiling the mechanism underlying the phenomena of resonance and cancellation of the system, as well as for the mitigation of vibrations of the system.

(2) The neglecting of the relative displacements between the wheel, track, and bridge may lead to an increase of the peak values of the PSD of the TSTBI system. Furthermore, the extent of the amplification for the lateral accelerations of the carbody is much larger than that for the vertical accelerations of the carbody.

(3) PEM may serve as a useful aid to the railway engineers for optimizing the design of dynamic parameters of the vehicle, track, and bridge to ensure the dynamic stability of a train traveling over a bridge under the actions of track irregularities and earthquakes on the basis of random vibration.

\section{Conflict of Interests}

The authors declare that there is no conflict of interests regarding the publication of this paper.

\section{Acknowledgments}

The research summarized in this paper was supported by the Joint Funds of the National Natural Science Foundation of China through Grants nos. U1334203 and U1361204, the National Natural Science Foundation of China through Grant no. 51378513, the National Key Technology R\&D Program of China through Grant no. 2013BAG20BH00, the Program for Changjiang Scholars and Innovative Research Team in University through Grant no. IRT1296, and the Science and Technology Foundation of China Railway Corporation through Grants nos. 2013G008-E and 2014G001-D. These supports are gratefully acknowledged. 


\section{References}

[1] S. H. Ju and H. C. Li, "Dynamic interaction analysis of trains moving on embankments during earthquakes," Journal of Sound and Vibration, vol. 330, no. 22, pp. 5322-5332, 2011.

[2] W. M. Zhai and C. B. Cai, “Train/track/bridge dynamic interactions: simulation and applications," Vehicle System Dynamics, vol. 37, supplement, pp. 653-665, 2002.

[3] R. M. Delgado and R. C. S. M. dos Santos, "Modelling of railway bridge-vehicle interaction on high speed tracks," Computers \& Structures, vol. 63, no. 3, pp. 511-523, 1997.

[4] L. K. Chen, L. Z. Jiang, and P. Liu, "Seismic response analyses of high-speed railway bridge round-ended piers using global bridge model," International Journal of Materials and Product Technology, vol. 44, no. 1-2, pp. 35-46, 2012.

[5] Y. Bin, G.-L. Dai, and H.-P. Zhang, "Beam-track interaction of high-speed railway bridge with ballast track," Journal of Central South University, vol. 19, no. 5, pp. 1447-1453, 2012.

[6] Z. C. Zhang, J. H. Lin, Y. H. Zhang, Y. Zhao, W. P. Howson, and F. W. Williams, "Non-stationary random vibration analysis for train-bridge systems subjected to horizontal earthquakes," Engineering Structures, vol. 32, no. 11, pp. 3571-3582, 2010.

[7] Y.-B. Yang and Y.-S. Wu, "Dynamic stability of trains moving over bridges shaken by earthquakes," Journal of Sound and Vibration, vol. 258, no. 1, pp. 65-94, 2002.

[8] C.-W. Kim and M. Kawatani, "Effect of train dynamics on seismic response of steel monorail bridges under moderate ground motion," Earthquake Engineering and Structural Dynamics, vol. 35, no. 10, pp. 1225-1245, 2006.

[9] M. Sogabe, M. Ikeda, and Y. Yanagisawa, "Train-running quality during earthquakes and its improvement for railway long span bridges," Quarterly Report of RTRI, vol. 48, no. 3, pp. 183-189, 2007.

[10] J. D. Yau, "Response of a train moving on multi-span railway bridges undergoing ground settlement," Engineering Structures, vol. 31, no. 9, pp. 2115-2122, 2009.

[11] P. Antolín, N. Zhang, J. M. Goicolea, H. Xia, M. Á. Astiz, and J. Oliva, "Consideration of nonlinear wheel-rail contact forces for dynamic vehicle-bridge interaction in high-speed railways," Journal of Sound and Vibration, vol. 332, no. 5, pp. 1231-1251, 2013.

[12] O. Caglayan, K. Ozakgul, O. Tezer, and E. Uzgider, "Evaluation of a steel railway bridge for dynamic and seismic loads," Journal of Constructional Steel Research, vol. 67, no. 8, pp. 1198-1211, 2011.

[13] M. A. Saadeghvaziri and A. R. Yazdani-Motlagh, "Seismic behavior and capacity/demand analyses of three multi-span simply supported bridges," Engineering Structures, vol. 30, no. 1, pp. 54-66, 2008.

[14] V. Bisadi, M. Head, and D. B. H. Cline, "Seismic effects of elevating bridges with steel pedestals in the southeastern United States," Engineering Structures, vol. 33, no. 12, pp. 3279-3289, 2011.

[15] S. Miura, "Deformation of track and safety of train in earthquake," Quarterly Report of RTRI, vol. 37, no. 3, pp. 139-147, 1996.

[16] T. Miyamoto, H. Ishida, and M. Matsuo, "Running safety of railway vehicle as earthquake occurs," Quarterly Report of RTRI, vol. 38, no. 3, pp. 117-122, 1997.

[17] Y. B. Yang, J. D. Yau, and Y. S. Wu, Vehicle-Bridge Interaction Dynamics: with Applications to High-Speed Railways, World Scientific Publishing, Singapore, 2004.
[18] N. Matsumoto, M. Sogabe, H. Wakui, and M. Tanabe, "Running safety analysis of vehicles on structures subjected to earthquake motion," Quarterly Report of RTRI, vol. 45, no. 3, pp. 116-122, 2004.

[19] N. Zhang, H. Xia, and G. D. Roeck, "Dynamic analysis of a train-bridge system under multi-support seismic excitations," Journal of Mechanical Science and Technology, vol. 24, no. 11, pp. 2181-2188, 2010.

[20] L. Gao, K.-M. Yin, and G.-Y. Zhang, "Study on dynamics characteristics of concrete floating slab track in urban track," Key Engineering Materials, vol. 302-303, pp. 700-705, 2006.

[21] T. Xin and L. Gao, "Reducing slab track vibration into bridge using elastic materials in high speed railway," Journal of Sound and Vibration, vol. 330, no. 10, pp. 2237-2248, 2011.

[22] D. Q. Li, D. Bilow, and T. Sussmann, "Slab track for shared freight and high speed passenger service," in Proceedings of the ASME Joint Rail Conference (JRC '10), vol. 1, pp. 41-47, Urbana, Ill, USA, April 2010.

[23] L.-K. Chen, L.-Z. Jiang, W. Guo, W.-S. Liu, Z.-P. Zeng, and G.W. Chen, "The seismic response of high-speed railway bridges subjected to near-fault forward directivity ground motions using a vehicle-track-bridge element," Shock and Vibration, vol. 2014, Article ID 985602, 17 pages, 2014.

[24] F.-S. Liu, Z.-P. Zeng, B. Wu, Z.-C. Zhang, and K. Peng, "Study of the effect of cement asphalt mortar disease on mechanical properties of CRTS II slab ballastless track," Advanced Materials Research, vol. 906, pp. 305-310, 2014.

[25] Z.-P. Zeng, Z.-W. Yu, Y.-G. Zhao, W.-T. Xu, L.-K. Chen, and P. Lou, "Numerical simulation of vertical random vibration of train-slab track-bridge interaction system by PEM," Shock and Vibration, vol. 2014, Article ID 304219, 21 pages, 2014.

[26] N. C. Nigam, Introduction to Random Vibration, MIT Press, Cambridge, Mass, USA, 1983.

[27] J. H. Lin, W. S. Zhang, and F. W. Williams, "Pseudo-excitation algorithm for non-stationary random seismic responses," Engineering Structures, vol. 16, no. 4, pp. 270-276, 1994.

[28] F. Lu, Q. Gao, J. H. Lin, and F. W. Williams, "Non-stationary random ground vibration due to loads moving along a railway track," Journal of Sound and Vibration, vol. 298, no. 1-2, pp. 3042, 2006.

[29] F. Lu, J. H. Lin, D. Kennedy, and F. W. Williams, "An algorithm to study non-stationary random vibrations of vehicle-bridge systems," Computers \& Structures, vol. 87, no. 3-4, pp. 177-185, 2009.

[30] P. Lou and Q.-Y. Zeng, "Formulation of equations of motion of finite element form for vehicle-track-bridge interaction system with two types of vehicles model," International Journal for Numerical Methods in Engineering, vol. 62, no. 3, pp. 435-474, 2005.

[31] P. Lou and F. T. K. Au, "Finite element formulae for internal forces of Bernoulli-Euler beams under moving vehicles," Journal of Sound and Vibration, vol. 332, no. 6, pp. 1533-1552, 2013.

[32] J. J. Kalker, On the Rolling Contact of Two Elastic Bodies in the Response of Dry Friction, Delft University of Technology, Delft, The Netherlands, 1967.

[33] N. Zhang, H. Xia, W. W. Guo, J. W. Zhan, J. B. Yao, and Y. M. Gao, "Vehicle-bridge interaction analysis of heavy load railway," Procedia Engineering, vol. 4, pp. 347-354, 2010.

[34] L. D. Zhu and Y. L. Xu, "Buffeting response of long-span cablesupported bridges under skew winds. Part 1: theory," Journal of Sound and Vibration, vol. 281, no. 3-5, pp. 647-673, 2005. 
[35] R. W. Clough and J. Penzien, Dynamics of Structures, McGrawHill, New York, NY, USA, 1993.

[36] G. Q. Li and Q. S. Li, Engineering Structure of Time-Varying Reliability Theory and Its Application, Science Press, Beijing, China, 2001, (Chinese).

[37] X. T. Du, Y. L. Xu, and H. Xia, "Dynamic interaction of bridgetrain system under non-uniform seismic ground motion," Earthquake Engineering and Structural Dynamics, vol. 41, no. 1, pp. 139-157, 2012.

[38] W. M. Zhai and H. Xia, Train-Track-Bridge Dynamic Interaction Theory and Engineering Application, Science Press, Beijing, China, 2011, (Chinese). 


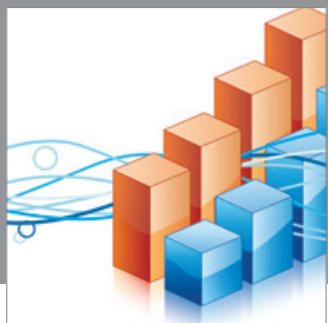

Advances in

Operations Research

mansans

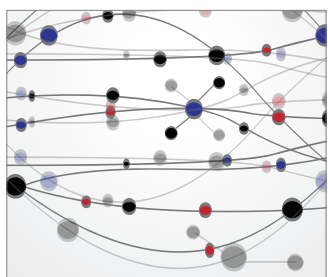

The Scientific World Journal
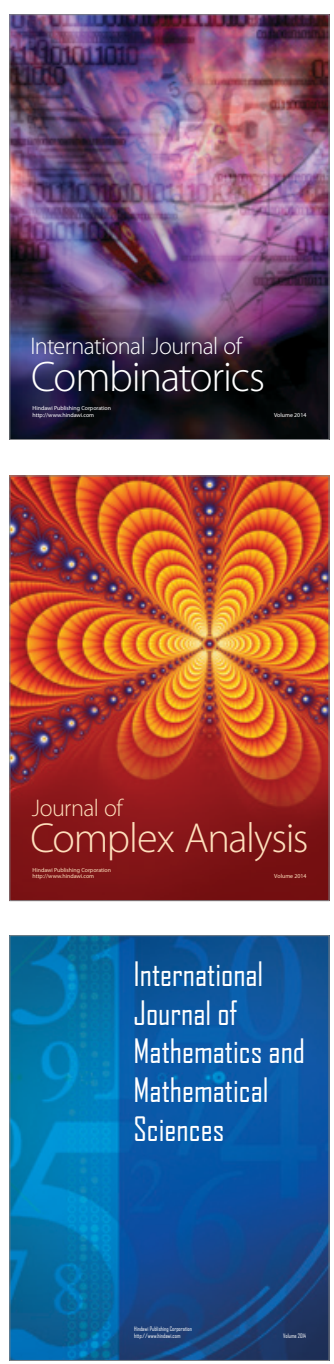
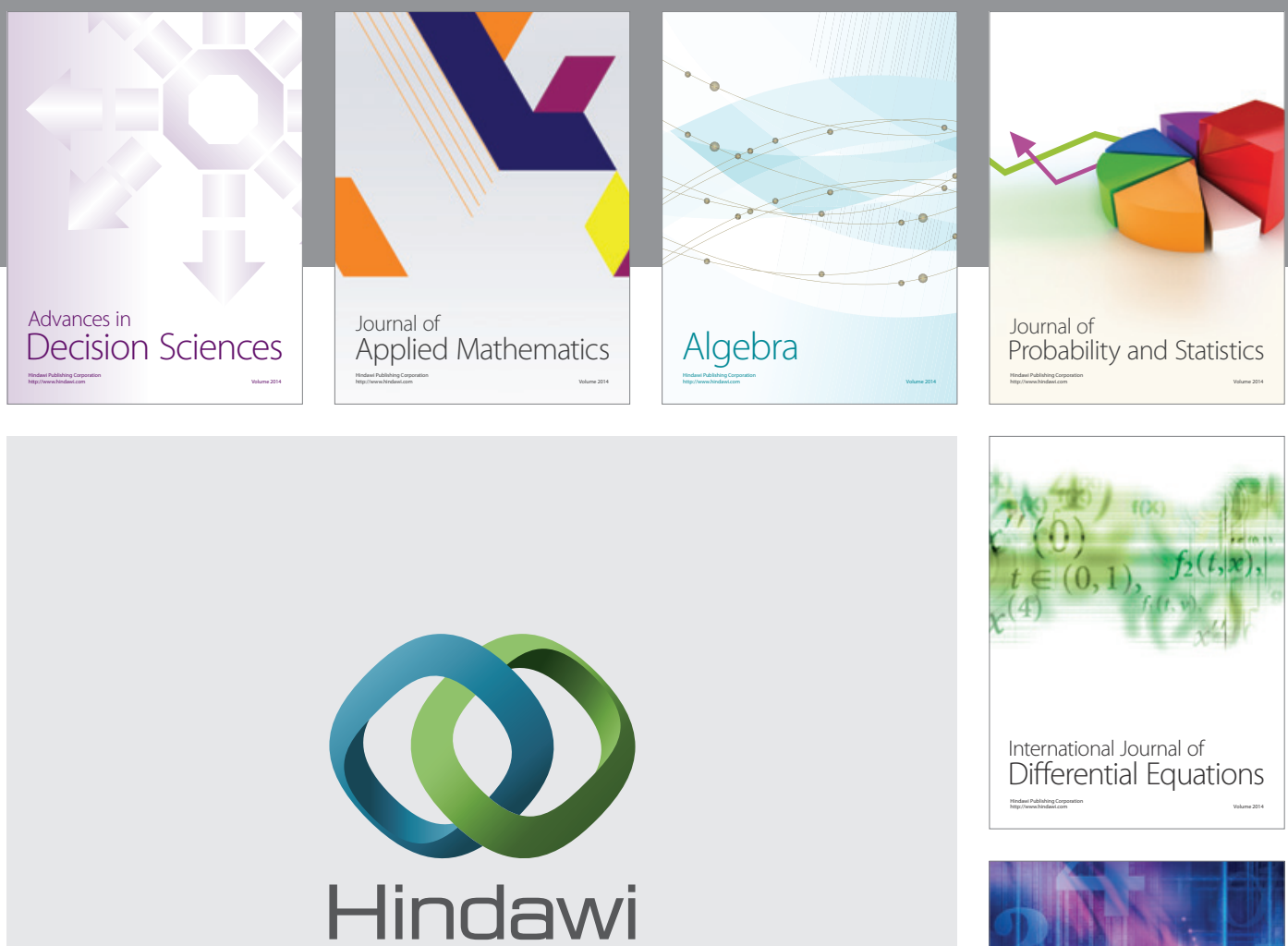

Submit your manuscripts at http://www.hindawi.com
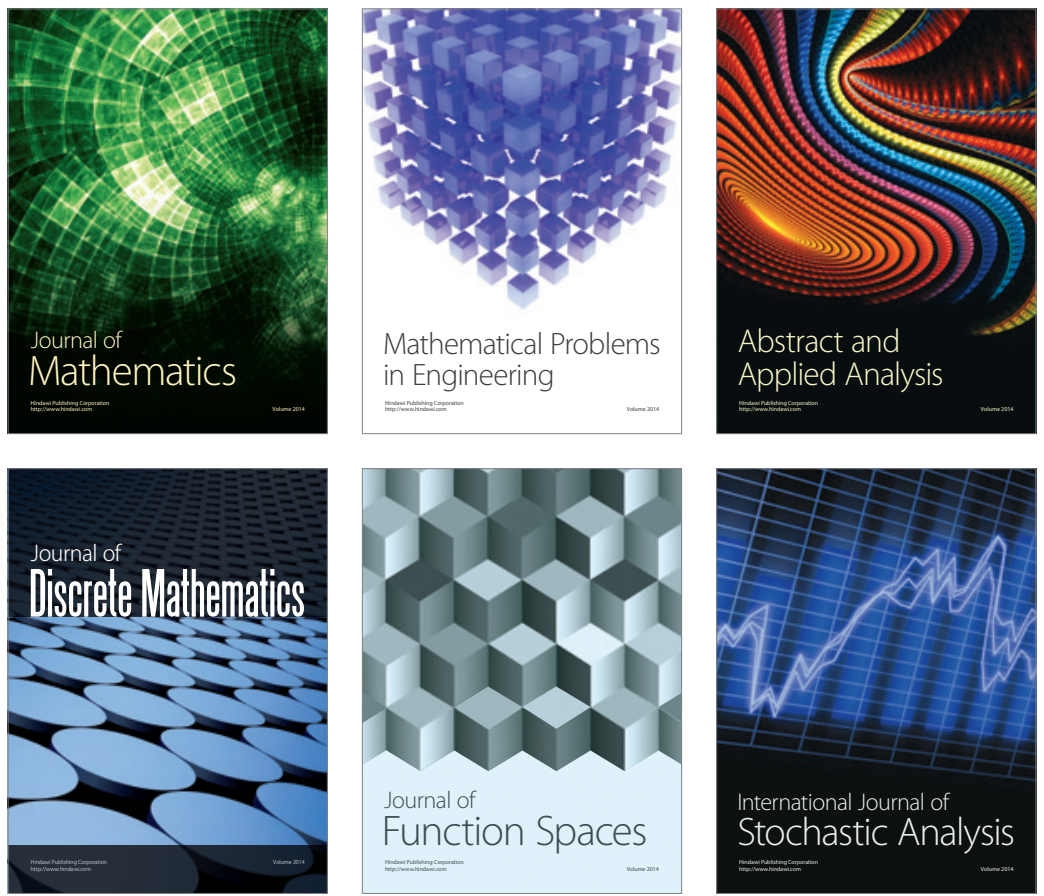

Journal of

Function Spaces

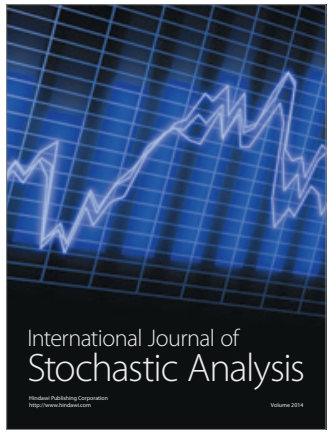

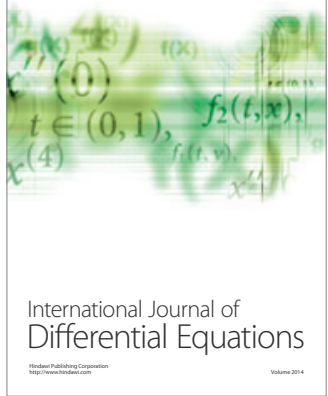
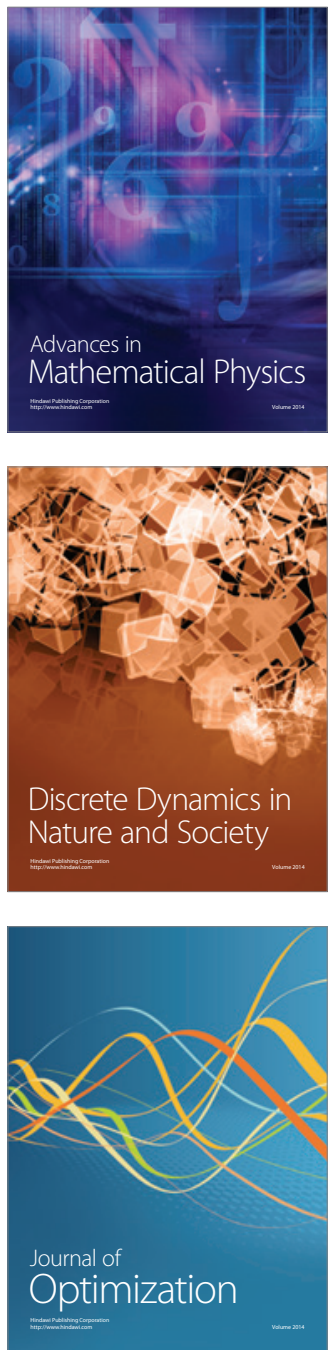